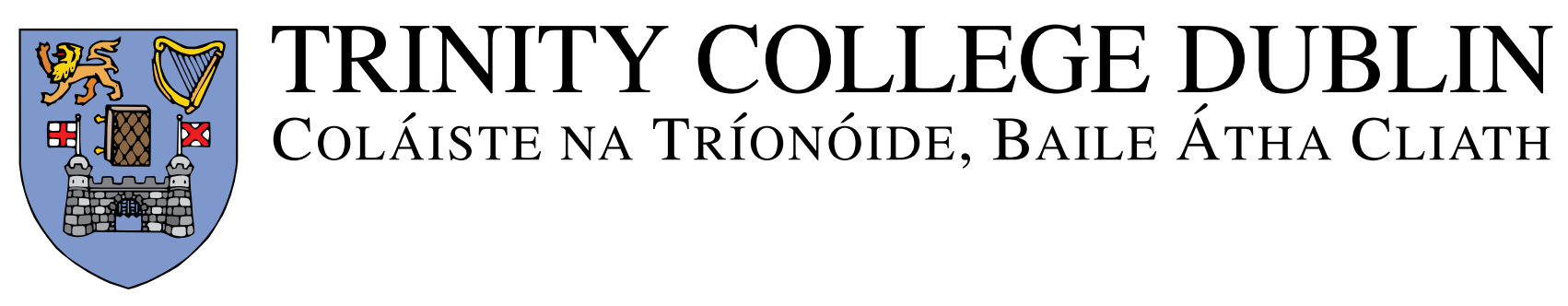

\title{
Modelling session types using contracts
}

Giovanni Bernardi and Matthew Hennessy 


\title{
Modelling session types using contracts
}

\author{
Giovanni Bernardi and Matthew Hennessy
}

August 2, 2011

\begin{abstract}
Session types and contracts are two formalisms used to study clientserver protocols. In this paper we study the relationship between them. The main result is the existence of a fully abstract model of session types; this model is based on a natural interpretation of these types into a subset of contracts 1
\end{abstract}

\section{Contents}

$\begin{array}{lll}1 & \text { Introduction } & 1\end{array}$

2 Session types 3

2.1 Sub-typing $\ldots \ldots \ldots \ldots \ldots \ldots \ldots \ldots$

\begin{tabular}{lll}
\hline 3 & Contracts & 8
\end{tabular}

3.1 The contract language $\ldots \ldots \ldots \ldots \ldots$

3.1.1 Client-Server interactions and the compliance relation . . 14

3.2 The server pre-order $\ldots \ldots \ldots \ldots \ldots \ldots$

$3.2 .1 \quad$ Co-inductive characterisation . . . . . . . . . . . . . . 19

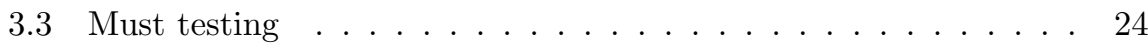

\begin{tabular}{|lll}
\hline & Session Contracts & 29
\end{tabular}

4.1 Session contracts . . . . . . . . . . . . . . . . . . . . . . 29

4.2 The server pre-order . . . . . . . . . . . . . . . . . . . 30

4.3 The client pre-order $\ldots \ldots \ldots \ldots \ldots$. . . . . . . . . . . 35

$\begin{array}{|lll|}5 & \text { Modelling session types } & 38\end{array}$

$5.1 \quad$ Examples and applications $\ldots \ldots \ldots \ldots \ldots$. . . . . . . . 41

6 Conclusions 43

6.1 Summary . . . . . . . . . . . . . . . . . . . 43

\section{Introduction}

Communication between processes in a distributed system often consists of a structured dialogue, following a protocol which specifies the format of the messages interchanged and, at least for binary communication, the direction of the

\footnotetext{
${ }^{1}$ This research was supported by SFI project 06 IN.1 1898.
} 
messages. Session types, $\mathcal{S T}$, have been introduced as an approach to the static analysis of the participants of such dialogues. They allow structured sequences of non-uniform messages to be interchanged between the participants. For example, using the notation of [GH05, the type ![Int]; ?[Real]; END specifies the output of a value of type Int followed by the input of a value of type Real, after which the dialogue is terminated. Flexibility in the permitted sequencing of messages by a process is accommodated by two choice operators; the branching type \& $\left\langle T_{1}, T_{2}\right\rangle$ offers a choice to the partner in the dialogue between following either the protocol specified by the type $T_{1}$ or that specified by $T_{2}$. On the other hand the choice type $\oplus\left\langle T_{1}, T_{2}\right\rangle$ allows the process itself to follow either of the protocols specified by $T_{1}$ or $T_{2}$.

Sub-typing, GH05, also increases the flexibility of the type system; intuitively $T_{1} \preccurlyeq_{\mathrm{ST}} T_{2}$ means that any participant designed with the protocol specified by $T_{1}$ in mind may also be used in a situation where the protocol specified by $T_{2}$ will be followed. Intuitively this pre-order between session types is generated by allowing more possibilities in branching types and restricting them in choice types. The reader is refered to HVK98, GH05, CP10 for more details on session types, including how they are associated with processes and what behaviour they guarantee.

Web services ACKM04, BPZ09 are distributed components which can be combined using standard communication protocols and machine-independent message formats to provide services to clients. To encourage reusability, descriptions of their behaviour are typically made available in searchable repositories OasisS11. In papers such as CCLP06, LP07, CGP09, Bd10 a language of contracts has been proposed for describing this behaviour which, despite a very different surface syntax, is very similar in style to session types. In particular there is the sequencing of messages $\alpha_{1} . \alpha_{2}$, an external choice between behaviours $\sigma_{1}+\sigma_{2}$ reminiscent of the branching type $\&\left\langle T_{1}, T_{2}\right\rangle$, and an internal choice between allowed behaviours $\sigma_{1} \oplus \sigma_{2}$, reminiscent of the choice type $\oplus\left\langle T_{1}, T_{2}\right\rangle$.

The object of this paper is to study the precise relationship between these two formalisms. In particular for first-order session types, which do not allow the use of communication channels in messages, we show that the theory of session types, $\left\langle\mathcal{S T}, \preccurlyeq_{\mathrm{sT}}\right\rangle$, can be captured precisely using a natural pre-order over a natural subclass of contracts.

Contracts for web services serve two roles. A contract $\sigma$ may describe the behaviour of a server offering some specific service. Dually a contract $\rho$ may describe the behaviour expected of a client who wishes to avail of a particular service. Central to the theory of contracts for web services is the idea of compliance between such contracts, formalised as an asymmetric relation $\rho \dashv \sigma$; it has been defined in a variety of ways in papers such as [LP07, LP08, CGP09. This leads to two natural pre-orders on contracts, defined set theoretically:

- the server pre-order: $\sigma_{1} \sqsubseteq_{\mathrm{SRV}} \sigma_{2}$ if for every (client) contract $\rho, \rho \dashv \sigma_{1}$ implies $\rho \dashv \sigma_{2}$

- the client pre-order: $\rho_{1} \sqsubseteq_{\text {СLt }} \rho_{2}$ if for every (server) contract $\sigma, \rho_{1} \dashv \sigma$ implies $\rho_{2} \dashv \sigma$

As we have already stated session types are more or less a syntactic variant of contracts; formally there is a straightforward translation $\mathcal{M}(T)$ of session types 
into contracts. Unfortunately neither of the relations $\sqsubseteq_{\mathrm{SRV}}$, $\sqsubseteq_{\mathrm{CLT}}$ are sound with respect to sub-typing; specifically there are session types $T_{1}, T_{2}$ such that $T_{1} \preccurlyeq_{\text {sT }} T_{2}$ but $\mathcal{M}\left(T_{1}\right)$ and $\mathcal{M}\left(T_{2}\right)$ are unrelated as contracts.

The problem lies in the fact that, viewed as constraints on behaviour, session types are much more constraining than contracts. We therefore isolate a subset of contracts, which we call session contracts $\mathcal{S C}$, which are the range of the translation function $\mathcal{M}$. This enables us to define more restrictive sub-server and sub-client relations, $\complement_{\mathrm{SRV}}^{\mathcal{S C}}$ and $\complement_{\mathrm{CLT}}^{\mathcal{S C}}$ respectively, on these contracts. It turns out that these relations are still unsound with respect to session subtyping. But in the main result of the paper we show that by combining these pre-orders we obtain full-abstraction, that is a sound and complete model for session types.

The paper is organised as follows. In the next section we give the definition of session types and the sub-typing between them; this material is taken directly from [GH05], although our definition is based on first-order types; however, we allow a primitive sub-typing relation between the basic types.

In the subsequent section we study contracts. Our language for contracts is a subset of the language proposed in [Pad10]; we provide a novel co-inductive formulation of the notion of compliance; nevertheless, the resulting compliance relation coincides with that used in both in [Pad10] and [Bd10]. The sub-typing relation between session types is defined co-inductively and the connection we eventually make between contracts and session types will depend on co-inductive characterisations of the set-based pre-orders on contracts. As an example of this we provide a co-inductive characterisation of the server pre-order on contracts; this definition is based on their behaviour. There is also much similarity between the idea of contract compliance and must-testing [NH84], as has been pointed out in LP07. We also prove that in our framework the must-testing pre-order over contracts coincides with the server pre-order $\sqsubseteq_{\mathrm{SRV}}$.

In Section 4 we focus on a subset of contracts called session contracts $\mathcal{S C}$, this time giving co-inductive characterisations to both the restricted server preorder $\sqsubseteq_{\mathrm{SRV}}^{\mathcal{S C}}$ and the restricted client pre-order $\sqsubseteq_{\mathrm{CLT}}^{\mathcal{S C}}$ over them. Due to the very restricted nature of these contracts, these co-inductive characterisations are purely in terms of their syntax.

In Section 5 we tackle the central question of the paper. Having defined the (obvious) translation of session types into session contracts, we explain why the two natural pre-orders $\varlimsup_{\mathrm{SRV}}^{\mathcal{S C}}$ and $\varlimsup_{\mathrm{CLT}}^{\mathcal{S C}}$ are unsound relative to the subtyping on session types. Finally, we prove that when combined they provide a sound and complete model; the proof is greatly facilitated by their co-inductive characterisations. The paper concludes with a brief look at related work.

\section{Session types}

The syntax of terms for session types is given by the language $L_{\mathcal{S T}}$ in Figure 1 . It presupposes a denumerable set of labels $\mathbb{L}$, ranged over by 1 , and a set of basic or ground types BT types ranged over by $t$. We also use a denumerable set of variables Vars, ranged over by $X$, in order to express recursive types.

The use of variables leads to the usual notion of free and bound occurrences of variables in terms in the standard manner; we say that a term is closed if it contains no free variables. We also have the standard notion of capture 


\begin{tabular}{|c|c|c|}
\hline$S, T \quad::=$ & $\begin{array}{l}\text { END } \\
?[\mathrm{t}] ; S \\
![\mathrm{t}] ; S \\
\&\left\langle\mathrm{I}_{1}: S_{1}, \ldots, \mathrm{I}_{n}: S_{n}\right\rangle, n \geq 1 \\
\oplus\left\langle\mathrm{I}_{1}: S_{1}, \ldots, \mathrm{I}_{n}: S_{n}\right\rangle, n \geq 1 \\
X \\
\mu X . S\end{array}$ & $\begin{array}{l}\text { Session types } \\
\text { satisfaction } \\
\text { input } \\
\text { output } \\
\text { branch } \\
\text { choice } \\
\text { type variable } \\
\text { recursion }\end{array}$ \\
\hline
\end{tabular}

We impose the additional proviso that in a term the $l_{i}$ 's are pair-wise different.

Figure 1: Session types (first-order).

$$
\frac{T}{T} T \neq \mu Z . S \quad \frac{T\{\mu Y \cdot T / Y\}}{\mu Y . T}
$$

Figure 2: Inference rules for $\downarrow_{\mathrm{DPT}}$ on closed terms.

avoidance substitution of terms for free variables. For the sake of clarity let us recall this definition: a substitution $\mathbf{s}$ is a mapping from the set Vars to the set of terms in $L_{\mathcal{S T}}$. Let

$$
\mathbf{s}-X= \begin{cases}\mathbf{s} \backslash\{(X, \mathbf{s}(X))\} & \text { if } X \in \operatorname{dom}(\mathbf{s}) \\ \mathbf{s} & \text { otherwise }\end{cases}
$$

Then the result of applying a substitution s to the term $S$ is defined as follows:

$$
S \mathbf{s}= \begin{cases}\mathrm{END} & \text { if } S=\mathrm{END} \\ \mathbf{s}(X) & \text { if } S=X, \text { and } X \in \operatorname{dom}(S) \\ X & \text { if } S=X, \text { and } X \notin \operatorname{dom}(S) \\ ![\mathrm{t}] ;\left(S^{\prime} \mathbf{s}\right) & \text { if } S=![\mathrm{t}] ; S^{\prime} \\ ?[\mathrm{t}] ;\left(S^{\prime} \mathbf{s}\right) & \text { if } S=?[\mathrm{t}] ; S^{\prime} \\ \&\left\langle 1_{1}:\left(S_{1} \mathbf{s}\right), \ldots, 1_{n}:\left(S_{n} \mathbf{s}\right)\right\rangle & \text { if } S=\&\left\langle 1_{1}: S_{1}, \ldots, 1_{n}: S_{n}\right\rangle \\ \oplus\left\langle 1_{1}:\left(S_{1} \mathbf{s}\right), \ldots, 1_{n}:\left(S_{n} \mathbf{s}\right)\right\rangle & \text { if } S=\oplus\left\langle 1_{1}: S_{1}, \ldots, 1_{n}: S_{n}\right\rangle \\ \mu X .\left(S^{\prime}(\mathbf{s}-X)\right) & \text { if } S=\mu X . S^{\prime}\end{cases}
$$

In the final clause the application of $\mathbf{s}-X$ embodies the idea that in $\mu X . S^{\prime}$ occurrences of $X$ in the sub-term $S^{\prime}$ are bound and therefore substitutions have no effect on them.

It is easy to check that the effect of a substitution depends only on free variables; that is, $S \mathbf{s}_{1}=S \mathbf{s}_{2}$ whenever $\mathbf{s}_{1}(X)=\mathbf{s}_{2}(X)$ for every free variable $X$ occurring in $S$. We use $\left\{{ }^{T} / X\right\}$ to denote the singleton substitution $\{(X, T)\}$.

We will only use guarded recursion, which we now explain formally. Let $\downarrow_{\mathrm{DPT}}$ be the least fixed point of the functor on closed terms defined by the inference rules in Figure 2 
Intuitively, $T \downarrow_{\mathrm{DPT}}$ means that the free variables in $T$ occur after a type constructor, which differs from $\mu$. Now we say that a term $T$ is guarded if every sub-term of the form $\mu X . S$ satisfies $S \downarrow_{\downarrow \text { ргт. }}$ Finally, we use $\mathcal{S T}$ to denote the set of closed guarded terms, and we refer to the elements in $\mathcal{S T}$ as session types.

Example 2.1. The property $\downarrow_{\mathrm{DPT}}$ and the property of being guarded are different. Consider the term $T=\&\langle 1: \mu X . X\rangle$; it is not a variable and the top-most constructor in it is not a recursion, therefore $T \downarrow_{\text {मPт }}$. A sub-term of $T$ is $\mu X . X$ and clearly $\mu X . X \downarrow_{\mathrm{DPT}}$ is false; therefore $T$ is not guarded.

The advantage of only using guarded terms is that we can unfold types so as to obtain their top-most type constructor. To explain this formally first let us consider the function $d p t$ from terms to $\mathbb{N}^{\infty}$ (the set of natural numbers augmented by $\infty$ ). This is defined as the least such function which satisfies:

$$
\operatorname{dpt}(S)= \begin{cases}1+\operatorname{dpt}\left(S^{\prime}\{S / X\}\right) & \text { if } S=\mu X . S^{\prime} \\ 0 & \text { otherwise }\end{cases}
$$

Note that $\operatorname{dpt}(\mu X . X)=\infty$, but one can show that when applied to terms that satisfy $\downarrow_{\mathrm{DPT}}$, one always obtains a natural number.

Lemma 2.2. If $S \downarrow_{\mathrm{DPT}}$ then $\operatorname{dpt}(S) \in \mathbb{N}$.

Proof. The proof is by rule induction on the derivation of $S \downarrow_{\text {DPT }}$.

If the axiom was used then thanks to the side condition $S \neq \mu X . S^{\prime}$, and so $\operatorname{dpt}(S)=0$ because of the definition of $d p t$. If the other rule was used then $S=\mu X . S^{\prime}$, and the hypothesis of the rule implies $S\left\{S^{\prime} / X\right\} \downarrow_{\mathrm{DPT}}$. Then, by definition of $d p t, \operatorname{dpt}(S)=1+\operatorname{dpt}\left(S\left\{S^{\prime} / x\right\}\right)$, and by the inductive hypothesis $\operatorname{dpt}\left(S\left\{S^{\prime} / X\right\}\right) \in \mathbb{N}$; hence $\operatorname{dpt}(S) \in \mathbb{N}$.

Proposition 2.3. The depth of any session type is finite.

Proof. Follows from the definition of $\mathcal{S T}$ and Lemma 2.2

This function $d p t$ will therefore provide a measure of session types over which we can perform induction.

Definition 2.4. [Unfolding GH05]]

For all $T \in \mathcal{S T}$, define $\operatorname{UNFOLD}(T)$ as follows:

$$
\operatorname{Unfold}(T)= \begin{cases}\operatorname{UNFOLD}\left(T^{\prime}\{\mu X . T / X\}\right) & \text { if } T=\mu X . T^{\prime} \\ T & \text { otherwise } \square\end{cases}
$$

Lemma 2.5. For every $T \in \mathcal{S T}$, $\operatorname{UNFOLD}(T)$ is a well-defined session type.

Proof. We have to show that $\operatorname{Unfold}(T)$ is closed and guarded. The proof is by induction on $\operatorname{dpt}(P)$. It relies on the fact that each step of unfolding replaces one variable with a closed and guarded term; hence the overall unfolding is closed. 


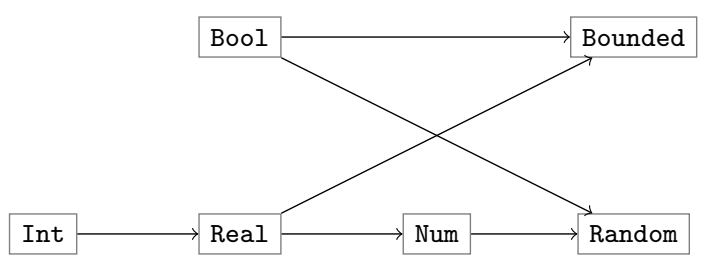

Figure 3: A sub-type relation on a set of basic types BT.

Intuitively, $\operatorname{UNFOLD}(T)$ unfolds top-level recursive definitions until a type constructor appears, which is not $\mu$. This will be extremely useful in manipulating session types.

We conclude this sub-section by showing some typical examples of session types, which we recall from the literature.

Example 2.6. [Math server, GH05]

Consider the session type

$$
\begin{array}{r}
S_{1}=\mu X . \&\langle\text { plus : ?[Int }] ; ?[\operatorname{Int}] ; ![\operatorname{Int}] ; X, \\
\text { eq }: ?[\operatorname{Int}] ; ?[\operatorname{Int}] ; ![\text { Bool }] ; \text { END }\rangle
\end{array}
$$

This specifies the protocol of a server which offers two services at the labels plus and eq. The first expects the input of two integers, after which an integer is returned, and then the service is once more available. The second also expects two integers, then returns a boolean, after which the session terminates.

An extension to the service is specified by the type

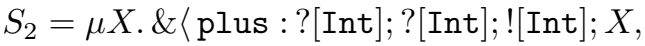

$$
\begin{aligned}
& \text { eq : ?[Int];?[Int];![Bool]; END, } \\
& \text { neg :?[Bool];![Bool]; END }\rangle
\end{aligned}
$$

This provides in addition queries for negation.

\section{$2.1 \quad$ Sub-typing}

There are three sources for the sub-typing relation over types. The first is some predefined pre-order over the basic types, $t_{1} \preccurlyeq_{\mathrm{g}} t_{2}$, which intuitively says that all data-values of type $t_{1}$ may be safely used where values of $t_{2}$ are expected. An example is given in Figure 3 , for the set of basic types $\mathrm{BT}=\{$ Bounded, Bool, Int, Real, Num, Random $\}$. More generally, if $\llbracket \mathrm{t} \rrbracket$ denotes the set of values of the basic type $t$ then we can define $\preccurlyeq_{\mathrm{g}}$ by letting $t_{1} \preccurlyeq_{\mathrm{g}} t_{2}$ whenever $\llbracket t_{1} \rrbracket \subseteq \llbracket t_{2} \rrbracket$. The other sources are two constructs of the language: the branch construct allows sub-typing by extending the set of labels involved, while in the choice construct the set of labels may be restricted. For example if $m \leq n$ we will have

$$
\begin{array}{rll}
\&\left\langle I_{1}: S_{1}, \ldots, I_{m}: S_{m}\right\rangle & \text { subtype of } & \&\left\langle I_{1}: S_{1}, \ldots, I_{n}: S_{n}\right\rangle \\
\oplus\left\langle I_{1}: S_{1}, \ldots, I_{n}: S_{n}\right\rangle & \text { subtype of } & \oplus\left\langle I_{1}: S_{1}, \ldots, I_{m}: S_{m}\right\rangle
\end{array}
$$


Moreover, we will have the standard co-variance/contra-variance of input/output types [PS96], extended to both the branch and choice constructs.

However, because of the recursive nature of our collection of types, the formal definition of the sub-typing relation is given co-inductively.

Definition 2.7. [Type simulation]

Let $\mathcal{P}(X)$ denote the powerset of a set $X$ and let $\mathcal{F}_{\preccurlyeq \text { sт }}: \mathcal{P}\left(\mathcal{S} \mathcal{T}^{2}\right) \longrightarrow \mathcal{P}\left(\mathcal{S} \mathcal{T}^{2}\right)$ be the function defined so that $(T, U) \in \mathcal{F}_{\preccurlyeq \text { ST }}(\mathcal{R})$ whenever one of the following holds:

1. if $\operatorname{UNFOLD}(T)=$ END then $\operatorname{UNFOLD}(U)=\mathrm{END}$

2. if $\operatorname{Unfold}(T)=$ ?[ $\left.\mathrm{t}_{1}\right] ; S_{1}$ then $\operatorname{Unfold}(U)=$ ?[ $\left[\mathrm{t}_{2}\right] ; S_{2}$ and $\left(S_{1}, S_{2}\right) \in \mathcal{R}$ and $\mathrm{t}_{1} \preccurlyeq \mathrm{g} \mathrm{t}_{2}$

3. if $\operatorname{UnFOLd}(T)=!\left[\mathrm{t}_{1}\right] ; S_{1}$ then $\operatorname{Unfold}(U)=$ ! $\left[\mathrm{t}_{2}\right] ; S_{2}$ and $\left(S_{1}, S_{2}\right) \in \mathcal{R}$ and $\mathrm{t}_{2} \preccurlyeq \mathrm{g} \mathrm{t}_{1}$

4. if $\operatorname{Unfold}(T)=\&\left\langle 1_{1}: S_{1}, \ldots l_{m}: S_{m}\right\rangle$ then $\operatorname{Unfold}(U)=\&\left\langle 1_{1}\right.$ : $\left.S_{1}^{\prime}, \ldots, 1_{n}: S_{n}^{\prime}\right\rangle$ where $m \leq n$ and $\left(S_{i}, S_{i}^{\prime}\right) \in \mathcal{R}$ for all $i \in[1, \ldots, m]$

5. if $\operatorname{Unfold}(T)=\oplus\left\langle\mathrm{l}_{1}: S_{1}, \ldots l_{m}: S_{m}\right\rangle$ then $\operatorname{Unfold}(U)=\oplus\left\langle 1_{1}\right.$ : $\left.S_{1}^{\prime}, \ldots, 1_{n}: S_{n}^{\prime}\right\rangle$ where $n \leq m$ and $\left(S_{i}, S_{i}^{\prime}\right) \in \mathcal{R}$ for all $i \in[1, \ldots, n]$

A relation $\mathcal{R}$ such that $\mathcal{R} \subseteq \mathcal{F}_{\preccurlyeq \text { ST }}(\mathcal{R})$ is called type simulation. The co-inductive sub-typing relation $\preccurlyeq_{\mathrm{ST}}$ is now defined as the greatest fixed point of the equation $X=\mathcal{F}_{\preccurlyeq \text { ST }}(X)$. Standard arguments ensure that the relation $\preccurlyeq_{\mathrm{ST}}$ exists, that it is a typing relation, and indeed the greatest type simulation.

Example 2.8. Let $T, S$ denote the types $\mu X$. ?[Int]; $X, \mu X$.?[Real]; $X$ respectively. Then $T \preccurlyeq_{\text {ST }} S$ because the relation $\mathcal{R}=\{(T, S),($ ? [nt]; $T$, ?[Real]; $S)\}$ is a type simulation, since Int $\preccurlyeq_{\mathrm{g}}$ Real.

Referring to Example 2.6 , one can also show that $S_{1} \preccurlyeq_{\mathrm{ST}} S_{2}$ by providing an appropriate type simulation.

The requirement that session types be guarded is crucial for the sub-typing relation to be well-defined. We explain this fact in the next example.

Example 2.9. [Sub-typing and guardedness]

Consider again the term $T=\&\langle 1: \mu X . X\rangle$ of Example2.1. Suppose we wanted to check whether

$$
T \preccurlyeq_{\mathrm{ST}} \&\langle 1: S\rangle
$$

for some session type $S$. The definition of $\preccurlyeq_{\mathrm{ST}}$ requires us to check whether $\operatorname{UNFOLD}(\mu X . X) \preccurlyeq_{\mathrm{ST}} \operatorname{UNFOLD}(S)$; this check, though, can not be done because

$$
\operatorname{UNFOLD}(\mu X . X)
$$

is not defined at all (and $\operatorname{UnFold}(S)$ may not be defined either).

Proposition 2.10. The relation $\preccurlyeq_{\mathrm{ST}}$ is a pre-order on $\mathcal{S T}$.

Proof. See GH05].

In GH05 the set of types $\mathcal{S} \mathcal{T}$ are used to give a typing system for the pi calculus, and appropriate Type Safety and Type Preservation theorems are proved. Here instead our aim is to give a model to the set of types $\left\langle\mathcal{S T}, \preccurlyeq_{\text {ST }}\right\rangle$ using contracts. 


$\begin{array}{cl}\sigma::= & \begin{array}{l}\text { Contracts } \\ \text { NIL }\end{array} \\ \mathbf{1} & \text { success } \\ \alpha . \sigma & \text { action } \\ \sigma+\sigma & \text { external choice } \\ \sigma \oplus \sigma & \text { internal choice } \\ x & \text { contract variable } \\ \mu x . \sigma & \text { recursion }\end{array}$

Figure 4: Contract grammar.

\section{Contracts}

We first define our language for contracts and give some examples. In the following sub-section we define a natural server based pre-order on contracts, for which we give a behavioural co-inductive characterisation. In the final subsection we investigate a closely related pre-order based on must testing.

\subsection{The contract language}

This subsection is roughly divided in three parts. In the first one we define the language $L_{\mathcal{C}}$, and we are concerned with syntactical properties of its terms $\sigma$ 's; similarly to what we have done for session types, we introduce the unfolding of closed terms of $L_{c}$ and a predicate $\downarrow_{\mathrm{DPT}}$ to guarantee that each contract can be unfolded (Lemma 3.1). Afterwards, we give an operational semantics (Figure 5), and we discuss important semantic properties that we want contracts to enjoy; this will lead to the introduction of a predicate $\downarrow$, and two lemmas (Lemma 3.10 and 3.11) which ensure that (a) contracts do not diverge, and (b) silent moves lead to a finite number of derivatives. In the last part of the subsection we describe how client-server interactions are modelled by contracts (Figure 7).

A language for contracts $L_{\mathcal{C}}$ is given in Figure 4 As with session types it uses a denumerable set of recursion variables Vars, here lower case, but also presupposes a set Act of actions, ranged over by $\alpha$, which processes guaranteeing contracts may perform; as we will see the special action $\checkmark$, which we assume is not in Act, will be used to indicate the fulfilment of a contract. Intuitively the contract $\alpha . \sigma$ performs the action $\alpha$ and then behaves like $\sigma$; the sum $\sigma^{\prime}+\sigma^{\prime \prime}$ is ready to behave either as $\sigma^{\prime}$ or as $\sigma^{\prime \prime}$ and the choice depends on the external environment. For this reason the operation + is called external sum. The internal sum $\sigma^{\prime} \oplus \sigma^{\prime \prime}$ represents a contract that can behave as $\sigma^{\prime}$ or as $\sigma^{\prime \prime}$, and the choice is taken by the contract independently from the environment. Such a decision can be due for instance to an IF statement in the process implementing the contracts. The symbol NIL denotes an empty contract, which intuitively can never be fulfilled, while $\mathbf{1}$ denotes the contract that is always satisfied.

Recursive definitions are handled in much the same way as session types and so we do not spell out the details; we assume a definition of capture-avoiding substitution s. Now we define the predicate $\downarrow_{\mathrm{DPT}}$, the function $d p t$, and the 
function UNFOLD as in the previous section.

The function $d p t$ is the least one that satisfies

$$
\operatorname{dpt}(\sigma)= \begin{cases}1+\operatorname{dpt}\left(\sigma^{\prime}\{\mu x . \sigma / x\}\right) & \text { if } \sigma=\mu x . \sigma^{\prime} \\ 0 & \text { otherwise }\end{cases}
$$

and the UNFOLD is the least function that satisfies:

$$
\operatorname{UNFOLD}(\sigma)= \begin{cases}\operatorname{UNFOLD}\left(\sigma^{\prime}\{\mu x \cdot \sigma / x\}\right) & \text { if } \sigma=\mu x \cdot \sigma^{\prime}, \\ \sigma & \text { otherwise }\end{cases}
$$

These definitions are not arbitrary. As it happens, they let us prove Lemma 5.2 . which, to our aim, is paramount (see Section 5).

Let $\downarrow_{\text {DPr }}$ be the least fixed point of the functor defined on closed terms of $L_{\mathcal{C}}$ by the rules in Figure 2

Similarly to what done in the previous section one can prove the following lemma.

Lemma 3.1. Let $\sigma \downarrow_{\text {DPT. }}$ Then

(i) the depth of $\sigma$ is finite: $\operatorname{dpt}(\sigma) \in \mathbb{N}$

(ii) the term $\operatorname{UNFOLD}(\sigma)$ is defined for and, if $\sigma$ is closed then $\operatorname{UNFOLD}(\sigma)$ is closed.

Proof. The proof of (ii) relies on (i) and is similar to the proof of Lemma 2.2 . Part (iii) can be proven by induction on $d p t$.

An operational semantics for the closed terms of the language $L_{\mathcal{C}}$ is given in Figure 5. The judgements are of the form

$$
\sigma \stackrel{\mu}{\longrightarrow} \sigma^{\prime}, \quad \mu \in A c t_{\tau} \checkmark
$$

where we use $A c t_{\tau} \checkmark$ as a shorthand for the set $A c t \cup\{\tau, \checkmark\}$. The judgement $\sigma \stackrel{\alpha}{\longrightarrow} \sigma$, where $\alpha \in A c t$ has the obvious meaning: $\sigma \stackrel{\tau}{\longrightarrow} \sigma^{\prime}$, means that the contract $\sigma$ is resolved to the contact $\sigma^{\prime}$ by some internal computation, while $\sigma \stackrel{\checkmark}{\longrightarrow} \sigma^{\prime}$ represents the reporting of the successful completion of a computation.

Let $\stackrel{\tau}{\longrightarrow}$ * denote the reflexive transitive closure of $\stackrel{\tau}{\longrightarrow}$.

We are now ready to show two properties of UNFOLD. We will use them we will require in the rest of the paper.

Lemma 3.2. Let $\sigma$ be a contract.

(i) If $\sigma \stackrel{\tau}{\nrightarrow}$ then $\operatorname{UNFOLD}(\sigma)=\sigma$

(ii) $\sigma \stackrel{\tau}{\longrightarrow} * \operatorname{UNFOLD}(\sigma)$

Proof. Property (i) is proved by structural induction on $\sigma$;

We prove part (iii); the argument is by induction on $\operatorname{dpt}(\sigma)$. If $\operatorname{dpt}(\sigma)=0$ then from the definition of $d p t$ it follows that $\sigma \neq \mu x . \sigma^{\prime}$; by definition of UNFOLD then $\operatorname{UNFOLD}(\sigma)=\sigma$. The reflexivity of $\stackrel{\tau}{\longrightarrow}^{*}$ implies $\sigma \stackrel{\tau}{\longrightarrow}{ }^{*} \operatorname{UNFOLD}(\sigma)$.

If $\operatorname{dpt}(\sigma)>1$ then, due to the definition of $d p t, \sigma=\mu x \cdot \sigma^{\prime}$. The definition of $\operatorname{UNFOLD}$ implies that $\operatorname{UNFOLD}(\sigma)=\operatorname{UNFOLD}\left(\sigma^{\prime}\{\sigma / x\}\right)$, while the definition of 


$$
\begin{aligned}
& {[\mathrm{A}-\mathrm{OK}]} \\
& \underset{\alpha . \sigma \stackrel{\alpha}{\longrightarrow} \sigma}{[\mathrm{A}-\mathrm{PRE}]} \\
& \overline{\mu x . \sigma \stackrel{\tau}{\longrightarrow} \sigma\{\mu x . \sigma / x\}}[\mathrm{A}-\mathrm{UNF}] \\
& \underset{\sigma \oplus \rho \stackrel{\tau}{\longrightarrow} \sigma}{[\mathrm{A}-\mathrm{IN}-\mathrm{L}]} \quad \underset{\sigma \oplus \rho \stackrel{\tau}{\longrightarrow} \rho}{[\mathrm{A}-\mathrm{IN}-\mathrm{R}]} \\
& \frac{\sigma \stackrel{\alpha}{\longrightarrow} \sigma^{\prime}}{\sigma+\rho \stackrel{\alpha}{\longrightarrow} \sigma^{\prime}}[\mathrm{R}-\mathrm{EXT}-\mathrm{L}] \quad \frac{\rho \stackrel{\alpha}{\longrightarrow} \rho^{\prime}}{\sigma+\rho \stackrel{\alpha}{\longrightarrow} \rho^{\prime}}[\mathrm{R}-\mathrm{EXT}-\mathrm{R}] \\
& \frac{\sigma \stackrel{\tau}{\longrightarrow} \sigma^{\prime}}{\sigma+\rho \stackrel{\tau}{\longrightarrow} \sigma^{\prime}+\rho}[\mathrm{R}-\mathrm{INT}-\mathrm{L}] \frac{\rho \stackrel{\tau}{\longrightarrow} \rho^{\prime}}{\sigma+\rho \stackrel{\tau}{\longrightarrow} \sigma+\rho^{\prime}}[\text { R-INT-R] }
\end{aligned}
$$

Figure 5: Inference rules for the semantics of closed terms of $L_{\mathcal{C}}$.

$d p t$ implies $\operatorname{dpt}(\sigma)=1+\operatorname{dpt}\left(\sigma^{\prime}\{\sigma / x\}\right)$, and therefore $\operatorname{dpt}\left(\sigma^{\prime}\{\sigma / x\}\right)$ is smaller than $\operatorname{dpt}(\sigma)$. We are now allowed to use the inductive hypothesis on $\sigma^{\prime}\left\{\sigma_{/ x}\right\}$ :

$$
\sigma^{\prime}\{\sigma / x\} \stackrel{\tau}{\longrightarrow}{ }^{*} \operatorname{UNFOLD}\left(\sigma^{\prime}\{\sigma / x\}\right)
$$

We use rule [A-UNF] (see Figure 5 to infer $\sigma \stackrel{\tau}{\longrightarrow} \sigma^{\prime}\{\sigma / x\}$, and then the transitivity of $\stackrel{\tau}{\longrightarrow}$ to obtain

$$
\sigma \stackrel{\tau}{\longrightarrow}{ }^{*} \operatorname{UNFOLD}\left(\sigma^{\prime}\left\{{ }^{\sigma} / x\right\}\right)
$$

We already know that $\operatorname{UNFOLD}(\sigma)=\operatorname{UNFOLD}\left(\sigma^{\prime}\{\sigma / x\}\right)$, and, by applying this equality to the reduction sequence above, we get

$$
\sigma \stackrel{\tau}{\longrightarrow}{ }^{*} \operatorname{UNFOLD}(\sigma)
$$

This concludes the proof.

Let

$$
\begin{aligned}
& S(\sigma)=\left\{\sigma^{\prime} \mid \sigma \stackrel{\mu}{\longrightarrow} \sigma^{\prime} \text { for some } \mu \in A_{c t} \checkmark\right\} \\
& F(\sigma)=\left\{\sigma^{\prime} \mid \sigma \stackrel{\tau}{\longrightarrow} \sigma^{\prime}\right\}
\end{aligned}
$$

One might think that $F(\sigma)$ is finite if and only if $\sigma$ does not diverge. This is not the case. 
Example 3.3. [Divergence and finite deriviatives]

Consider the terms $\mu x . x$ and $\mu x$. (NIL $\oplus x)$. Both terms diverge, in the sense that they perform an infinite sequence of $\tau$ 's:

$\mu x . x \stackrel{\tau}{\longrightarrow} \mu x . x, \quad \mu x .(\mathrm{NIL} \oplus x) \stackrel{\tau}{\longrightarrow} \mathrm{NIL} \oplus \mu x .(\mathrm{NIL} \oplus x) \stackrel{\tau}{\longrightarrow} \mu x .(\mathrm{NIL} \oplus x)$

On the other hand we have

$$
F(\mu x . x)=S(\mu x . x)=\{\mu x . x\}
$$

and

$$
\begin{aligned}
& F(\mu x .(\operatorname{NIL} \oplus x))=\{\mu x .(\operatorname{NIL} \oplus x), \operatorname{NIL} \oplus \mu x .(\operatorname{NIL} \oplus x)\} \\
& S(\mu x .(\operatorname{NIL} \oplus x))=\{\operatorname{NIL} \oplus \mu x .(\operatorname{NIL} \oplus x)\}
\end{aligned}
$$

The set $F(\sigma)$ is not finite for every $\sigma$.

Example 3.4. [Infinite derivatives]

We show two terms $\sigma, \sigma^{\prime}$ such that $F(\sigma)$ and $F(\sigma)$ are infinite. Let $\sigma=\mu x . \alpha . x+$ $x$ and $\sigma^{\prime}=\mu x$. $(\alpha . \mathrm{NIL}+(x \oplus x))$. Then according to the rules in Figure 5 one can infer:

$$
\sigma \stackrel{\tau}{\longrightarrow}(\alpha . \sigma+\sigma) \stackrel{\tau}{\longrightarrow}(\alpha . \sigma+(\alpha . \sigma+\sigma)) \stackrel{\tau}{\longrightarrow} \ldots
$$

and

$\sigma^{\prime} \stackrel{\tau}{\longrightarrow} \alpha . \mathrm{NIL}+\left(\sigma^{\prime} \oplus \sigma^{\prime}\right) \stackrel{\tau}{\longrightarrow} \alpha . \mathrm{NIL}+\sigma^{\prime} \stackrel{\tau}{\longrightarrow} \alpha . \mathrm{NIL}+\left(\alpha . \mathrm{NIL}+\left(\sigma^{\prime} \oplus \sigma^{\prime}\right)\right) \stackrel{\tau}{\longrightarrow} \ldots$

The root of the problem is that the inference rules [R-INT-L] and [R-INT-R] do not resolve the external sum.

On the other hand the finiteness of $S(\sigma)$ is easy to prove.

Lemma 3.5. [Finite branches]

The set $S(\sigma)$ is finite for every $\sigma$.

Proof. The proof is by structural induction. If $\sigma=$ NIL Then plainly $S(\sigma)=\emptyset$. Otherwise we proceed as follows.

- If $\sigma=\alpha \cdot \sigma^{\prime}$ then the only applicable rule (see Figure 5 is [A-PRE], and so $S(\sigma)=\left\{\sigma^{\prime}\right\}$

- similarly if $\sigma=\mu x . \sigma^{\prime}$. The only rule [A-UnF] can be applied, so $S(\sigma)=$ $\left\{\sigma^{\prime}\{\sigma / x\}\right\}$.

- If $\sigma=\sigma^{\prime}+\sigma^{\prime \prime}$ then it $\sigma^{\prime}$ and $\sigma^{\prime \prime}$ are both smaller than $\sigma$. The inductive hypothesis tells us that $S\left(\sigma^{\prime}\right)$ and $S\left(\sigma^{\prime \prime}\right)$ are finite. Rules [R-EXT-L], [REXT-R], [R-INT-L] and [R-INT-R] in Figure 5 ensure that $S(\sigma)=S\left(\sigma^{\prime}\right) \cup$ $S\left(\sigma^{\prime \prime}\right)$. This implies that the cardinality of $S(\sigma)$ is finite.

- If $\sigma=\sigma^{\prime} \oplus \sigma^{\prime \prime}$ the argument is alike the previous one. 


$$
\overline{\mathbf{1}} \quad \overline{\mathrm{NIL}} \quad \overline{\alpha . \sigma} \quad \frac{\rho \sigma}{(\sigma \oplus \rho)} \quad \frac{\rho \quad \sigma}{(\rho+\sigma)} \quad \frac{\sigma\{\mu x . \sigma / x\}}{\mu x . \sigma}
$$

Figure 6: Inference rules for $\downarrow$ over closed terms of $L_{\mathcal{C}}$.

Example 3.6. [Divergence and $\downarrow_{\mathrm{DPT}}$ ]

Consider the term

$$
\sigma=\mu x .(x \oplus x)
$$

Using the rules in Figure 2 one can prove that $\sigma \downarrow_{\mathrm{DPF}}$; consequently $\operatorname{dpt}(\sigma)$ is finite and $\operatorname{UNFOLD}(\sigma)$ is well-defined; in particular $\operatorname{dpt}(\sigma)=1$ and $\operatorname{UNFOLD}(\sigma)=$ $\sigma \oplus \sigma$. Note now that the term $\sigma$ engages in an infinite sequence of internal moves

$$
\sigma \stackrel{\tau}{\longrightarrow} \sigma \oplus \sigma \stackrel{\tau}{\longrightarrow} \sigma \stackrel{\tau}{\longrightarrow} \sigma \oplus \sigma \stackrel{\tau}{\longrightarrow} \ldots
$$

In other words the term $\sigma$ diverges. Similarly, one can reason that terms as $\mu x$. (NIL $\oplus x)$ and $\mu x .(\alpha \oplus x)$ suffer the same issue.

Throughout the paper we want to deal only with terms that do not diverge and with finite $F(\sigma)$. Unfortunately, $\downarrow_{\mathrm{DPT}}$ is too weak a predicate to force the terms that satisfy it to converge. To isolate the $\sigma$ 's that converge we use the predicate $\downarrow$. Formally, we define it as the least fixed point of the functor given by the inference rules in Figure 6. The predicate $\downarrow$ is essentially a strengthened version of $\downarrow_{\text {DPp. }}$.

Proposition 3.7. For every $\sigma \in L_{\mathcal{C}}, \sigma \downarrow$ implies $\sigma \downarrow_{\downarrow_{\mathrm{DPT}}}$.

Proof. Straightforward from the definitions of the predicates.

The predicate $\downarrow$ is preserved by silent moves.

Lemma 3.8. Let $\sigma \downarrow$. If $\sigma \stackrel{\tau}{\longrightarrow} \sigma^{\prime}$ then $\sigma^{\prime} \downarrow$.

Proof. The proof proceeds by rule induction on the derivation of $\sigma \stackrel{\tau}{\longrightarrow} \sigma^{\prime}$.

The only interesting case is when the silent move $\sigma \stackrel{\tau}{\longrightarrow} \sigma^{\prime}$ is inferred by using rule [R-ExT-L] or rule [R-ExT-R] (see Figure 5). Suppose rule [R-ExT-L] was used. Then $\sigma=\sigma_{1}+\sigma_{2}, \sigma^{\prime}=\sigma_{1}^{\prime}+\sigma_{2}$, and the derivation is

$$
\frac{\sigma_{1} \stackrel{\tau}{\longrightarrow} \sigma_{1}^{\prime}}{\sigma_{1}+\sigma_{2} \stackrel{\tau}{\longrightarrow} \sigma_{1}^{\prime}+\sigma_{2}}
$$

We have to prove that $\sigma_{1}^{\prime}+\sigma_{2} \downarrow$; the definition of $\downarrow$ ensures that, to this aim, it is enough to show that (a) $\sigma_{1}^{\prime} \downarrow$ and that (b) $\sigma_{2} \downarrow$ (see Figure 6). Point (b) follows from the hypothesis: since $\sigma \downarrow$, the equality $\sigma=\sigma_{1}+\sigma_{2}$ implies that $\sigma_{1} \downarrow$ and that $\sigma_{2} \downarrow$. The last fact is exactly point (b).

Now, by using the fact that $\sigma_{1} \downarrow$, we prove point (a). The derivation shown above let us use rule induction; the lemma holds for $\sigma_{1}$ : if $\sigma_{1} \downarrow$ and $\sigma_{1} \stackrel{\tau}{\longrightarrow} \hat{\sigma}_{1}$ then $\hat{\sigma}^{\prime} \downarrow$. We know that $\sigma_{1} \downarrow$ and that $\sigma_{1} \stackrel{\tau}{\longrightarrow} \sigma_{1}^{\prime}$, thus it must be $\sigma_{1}^{\prime} \downarrow$.

If rule [R-EXT-R] was used the argument is similar. 
We have also the converse.

Lemma 3.9. Let $\sigma$ be a closed term of $L_{\mathcal{C}}$. Then $\sigma \downarrow$ if and only if $\sigma \stackrel{\tau}{\longrightarrow} \sigma^{\prime}$ implies $\sigma^{\prime} \downarrow$.

Proof. The only if side of the lemma is Lemma 3.8 , so we have to prove only that if $\sigma \stackrel{\tau}{\longrightarrow} \sigma^{\prime}$ implies $\sigma^{\prime} \downarrow$, then $\sigma \downarrow$.

Let $\sigma$ be a closed term of $L_{\mathcal{C}}$ such that $\sigma \stackrel{\tau}{\longrightarrow} \sigma^{\prime}$ implies $\sigma^{\prime} \downarrow$. We have to show that $\sigma \downarrow$.

The proof is by structural induction on the form of $\sigma$; the only interesting case is when $\sigma$ is an external sum. In that case, $\sigma=\sigma_{1}+\sigma_{2}$, so to prove that $\sigma_{1} \downarrow$ it is enough to show that $\sigma_{1} \downarrow$ and that $\sigma_{2} \downarrow$.

We prove $\sigma_{1} \downarrow$. The term $\sigma_{1}$ is a sub-term of $\sigma$, hence structural induction guarantees that the lemma holds for $\sigma_{1}$ : if $\sigma_{1} \stackrel{\tau}{\longrightarrow} \sigma_{1}^{\prime}$ implies $\sigma_{1}^{\prime} \downarrow$, then $\sigma_{1} \downarrow$. Assume that $\sigma_{1} \stackrel{\tau}{\longrightarrow} \sigma_{1}^{\prime}$; thanks to the structure of $\sigma$ we can derive

$$
\frac{\sigma_{1} \stackrel{\tau}{\longrightarrow} \sigma_{1}^{\prime}}{\sigma_{1}+\sigma_{2} \stackrel{\tau}{\longrightarrow} \sigma_{1}^{\prime}+\sigma_{2}}[\text { R-EXT-L] }
$$

Now the hypothesis of the lemma implies that $\sigma_{1}^{\prime}+\sigma_{2} \downarrow$, so from the the definition of $\downarrow$ it follows that $\sigma_{1}^{\prime} \downarrow$ and that $\sigma_{2} \downarrow$.

We have shown that $\sigma_{1} \stackrel{\tau}{\longrightarrow} \sigma_{1}^{\prime}$ implies $\sigma_{1}^{\prime} \downarrow$, so from the inductive hypothesis it follows that $\sigma_{1} \downarrow$. Moreover, we have also shown that $\sigma_{2} \downarrow$; we have proven that $\sigma \downarrow$.

The for rule [R-EXT-L] is analogous, and left to the reader.

The predicate $\downarrow$ let us give an inductive characterisation of the convergent terms.

Lemma 3.10. [Convergence]

Let $\sigma$ be a closed term of $L_{\mathcal{C}}$. Then $\sigma \downarrow$ if and only if there exists a $k \in \mathbb{N}$ such that $\sigma \stackrel{\tau}{\longrightarrow}{ }^{n} \sigma^{\prime}$ implies $n \leq k$.

Proof. The only if side is by rule induction on why $\sigma \downarrow$.

We prove the if side, which states that if there exists a $k \in \mathbb{N}$ such that $\sigma \stackrel{\tau}{\longrightarrow}{ }^{n}{ }^{n} \sigma^{\prime}$ implies $n \leq k$, then $\sigma \downarrow$.

The argument is an induction on $k$. If $k=0$ then $\sigma$ can not perform $\tau$, and so it is either 1 , NIL or a prefix $\alpha . \sigma^{\prime}$. In all these cases we can easily infer $\sigma \downarrow$.

If $k>0$ then $\sigma$ performs a $\tau$, so suppose $\sigma \stackrel{\tau}{\longrightarrow} \sigma^{\prime}$; it follows that $\sigma^{\prime} \stackrel{\tau}{\longrightarrow}{ }^{*}{ }^{m} \sigma^{\prime \prime}$ implies $m \leq k-1$. This means that there exists a $k^{\prime}$ such that $\sigma^{\prime} \stackrel{\tau}{\longrightarrow}{ }^{*}{ }^{m} \sigma^{\prime \prime}$ implies $m \leq k^{\prime}$. Since $k-1<k$, we can apply the inductive hypothesis to $\sigma^{\prime}$; from this application it follows that $\sigma^{\prime} \downarrow$.

As yet, we have proven that $\sigma \stackrel{\tau}{\longrightarrow} \sigma^{\prime}$ implies $\sigma^{\prime} \downarrow$; this allows us to apply Lemma 3.9, which ensures that $\sigma \downarrow$.

It is easy to see that a converging term $\sigma$ has finite $F(\sigma)$.

Lemma 3.11. For every $\sigma \in L_{\mathcal{C}}$ if $\sigma \downarrow$ then $F(\sigma)$ is finite.

Proof. We can prove it by rule induction on the derivation of $\sigma \downarrow$. 
Now we let $\mathcal{C}$ denote the set of all terms $\sigma$ of $L_{\mathcal{C}}$ which are closed and satisfy $\downarrow$. We refer to these terms as contracts.

Proposition 3.12. Let $\sigma$ be a contract, then $\operatorname{UNFOLD}(\sigma)$ is a well-defined contract.

Proof. We have to show that $\operatorname{Unfold}(\sigma)$ is closed, and that it satisfies $\downarrow$. The first fact is part (iii) of Lemma 3.1. The second fact follows from part (ii) of Lemma 3.2 and Lemma 3.8

Example 3.13. [e-vote, [LP08, Bd10] ]

$$
\begin{aligned}
& \text { Ballot }=\mu x . \text { ?Login. }(\text { !Wrong } \cdot x \oplus \text { !ok. }(\text { ?VoteA } . x+\text { ?VoteB } . x)) \\
& \text { Voter }=\mu x . \text { !Login. }(\text { ?Wrong } \cdot x+\text { ?0k. }(\text { !VoteA.1 } \oplus \text { !VoteB.1 }))
\end{aligned}
$$

A process offering contract Ballot implements a service for e-voting. Such a service lets a client $\log$ in. If the $\log$ in fails the services starts anew, while if the $\log$ in succeeds the two actions are offered to the environment, namely VoteA and VoteB.

The contract Voter is a recursive client for the protocol described by the contract Ballot.

Example 3.14. [e-commerce, BBMR08]

$$
\begin{aligned}
& \text { Customer }=\text { !Request.(!PayDebit. } \rho^{\prime} \oplus \\
& \text { !PayCredit. } \rho^{\prime} \oplus \\
& \text { !PayCash.1) } \\
& \rho^{\prime} \quad=\text { !Long.?Bool.1 } \\
& \text { Bank }=\mu x \text {.?Request.(?PayCredit.?Long.!Bool. } x+ \\
& \text { ?PayDebit.?Long.!Bool. } x+ \\
& \text { ?PayCash. } x \text { ) }
\end{aligned}
$$

The contracts above describe the conversation that should take place between a client (which offers the contract Customer) and a bank (which has contract Bank) involved in an on-line payment. The conversation unfolds as follows: the Customer sends a request to the bank and afterwards it chooses the payment method; the choice is taken by an internal sum and this means that the decision of the Customer is independent from the environment (ie. the Bank contract). If the Customer decides to pay by cash then no other action has to be taken; while if the payment is done by debit or credit card the Customer has to send the card number, this is represented by the output !Long. After the card number has been received the Bank answers with a boolean. Intuitively, this represents the fact that the bank can approve or reject the payment. The Customer protocol finishes after such boolean has been received, while the Bank starts anew.

\subsubsection{Client-Server interactions and the compliance relation}

Contracts are expressive enough to encode XML based languages such as WSBPEL activities and WSCL diagrams CGP09. and in CCLP06 it is shown 
how to assign contracts to a subset of CCS processes. Intuitively, if a process, such as a server, is assigned a contract $\sigma$ then it guarantees to support the behaviour described in $\sigma$. The interaction between servers and clients can be described at the level of their contracts, by defining a binary operation $\rho \| \sigma$ between their contracts and describing the evolution of the contracts as they interact. This interacting semantics is given in Figure 7] where the judgements are of the form $\rho\left\|\sigma \stackrel{\tau}{\longrightarrow} \rho^{\prime}\right\| \sigma^{\prime}$. It presupposes a binary relation on Act, $\alpha \bowtie \beta$, which intuitively means that the action $\alpha$ can synchronise with action the $\beta$. This relation can be instantiated in various ways depending on the particular set of actions Act; the only general property we require of it is that it be finitary, that is for every action $\alpha$ the set $\{\beta \mid \alpha \bowtie \beta\}$ be finite.

Example 3.15. Suppose we take Act to be $\{a$ ?, $a$ ! $\mid a \in A\}$ where $A$ is a set of communicating channels. Then define

$\alpha \bowtie_{i} \quad \beta \quad$ whenever $\alpha=a$ ?, $\beta=a$ ! or $\alpha=a$ !, $\beta=a$ ? for some $a \in A$

This represents synchronisation on channels.

We will use a more elaborate set of actions when interpreting session types as contracts. Recall from Section 2 the set of basic types BT and the set of labels $\mathbb{L}$ used in session types. Using these we can define Act to be

$$
\{? \mathrm{~b}, \mathrm{l} \mathrm{b} \mid \mathrm{b} \in \mathrm{BT}\} \cup\{1 ?, \mathrm{l} ! \mid 1 \in \mathbb{L}\}
$$

with $\bowtie_{c}$ determined by

$$
\alpha \bowtie_{c} \beta \quad \text { whenever } \begin{cases}\alpha=? \mathrm{~b}, \beta=! \mathrm{b}^{\prime} & \mathrm{b}^{\prime} \preccurlyeq_{\mathrm{g}} \mathrm{b} \\ \alpha=! \mathrm{b}, \beta=? \mathrm{~b}^{\prime} & \mathrm{b} \preccurlyeq_{\mathrm{g}} \mathrm{b}^{\prime} \\ \alpha=? 1, \beta=! 1 & \\ \alpha=! 1, \beta=? 1 & \end{cases}
$$

Using the basic sub-typing relation depicted in Figure 3 the following examples should be clear:

(i) ?Num $\bowtie_{c}$ ! Int: a contract that can read a datum of type Num can read a datum of type Int because Int $\preccurlyeq \mathrm{g}$ Num.

(ii) ?Int $\not_{c}$ !Num: conversely a contract ready to read a datum of type Int can not read a datum of type Num because Num $\Varangle_{\mathrm{g}}$ Int.

(iii) ?Random $\bowtie_{c}$ ! Bool: as in point (i), Bool $\preccurlyeq_{\mathrm{g}}$ Random hence an interaction between the actions ?Random and !Bool is safe.

Having described how interactions between clients and servers affect their contracts, let us describe, by means of a relation, when a client (guaranteeing a) contract $\rho$ can safely interact with a server (guaranteeing a) contract $\sigma$. Indeed, we shall formalise the meaning of "safely".

The central notion is that of compliance between contracts. This is defined co-inductively and uses the predicate on contracts $\rho \stackrel{\checkmark}{\longrightarrow}$ which intuitively means that the contract $\rho$ has already been satisfied. Our definition is a variation on that of compliance in [LP07, LP08, Pad10]. 


$$
\begin{aligned}
& \frac{\rho \stackrel{\tau}{\longrightarrow} \rho^{\prime}}{\rho\left\|\sigma \stackrel{\tau}{\longrightarrow} \rho^{\prime}\right\| \sigma}[\mathrm{P}-\text { SIL-L }] \underset{\frac{\sigma}{\longrightarrow} \sigma^{\prime}}{\rho\|\sigma \stackrel{\tau}{\longrightarrow} \rho\| \sigma^{\prime}}[\text { P-SIL-R] } \\
& \frac{\rho \stackrel{\alpha}{\longrightarrow} \rho^{\prime} \quad \sigma \stackrel{\beta}{\longrightarrow} \sigma^{\prime}}{\rho\left\|\sigma \stackrel{\tau}{\longrightarrow} \rho^{\prime}\right\| \sigma^{\prime}} \alpha \bowtie \beta[\mathrm{P}-\mathrm{SYNCH}]
\end{aligned}
$$

Figure 7: Inference rules for contract interaction.

Definition 3.16. [Compliance relation]

Let $\mathcal{F}_{\dashv}: \mathcal{P}\left(\mathcal{C}^{2}\right) \longrightarrow \mathcal{P}\left(\mathcal{C}^{2}\right)$ be the function defined so that $(\rho, \sigma) \in \mathcal{F}_{\dashv}(\mathcal{R})$ whenever both the following hold:

(i) if $\rho \| \sigma \stackrel{\tau}{\longrightarrow}$ then $\rho \stackrel{\checkmark}{\longrightarrow}$

(ii) if $\rho\left\|\sigma \stackrel{\tau}{\longrightarrow} \rho^{\prime}\right\| \sigma^{\prime}$ then $\left(\rho^{\prime}, \sigma^{\prime}\right) \in \mathcal{R}$

If $\mathcal{R} \subseteq \mathcal{F}_{\dashv}(\mathcal{R})$ then we say that $\mathcal{R}$ is a co-inductive compliance relation. Let $\dashv$ denote the greatest solution of the equation $X=\mathcal{F}_{\dashv}(X)$. We call this solution the compliance relation. The relation $\dashv$ is the greatest co-inductive compliance relation. If $\rho \dashv \sigma$ we say that the contract $\rho$ complies with the contract $\sigma$.

Notice that there is an asymmetry in the relation $\rho \dashv \sigma$; the intention is that any client running contract $\rho$ when interacting with a server running contract $\sigma$ will be satisfied, in the sense that either the interaction between client and server will go on indefinitely, or, if the interaction gets stuck, the client will end on its own in a state in which it is satisfied, $\rho \stackrel{\checkmark}{\longrightarrow}$.

Example 3.17. [Compliance and divergent terms]

In order that the relation $\dashv$ captures the intuition described above, it is crucial that $\mathcal{C}$ contains no divergent terms. Had we admitted them, then for every $\rho$ the relation

$$
\left\{\left(\rho^{\prime}, \mu x . x\right) \mid \rho \stackrel{\tau}{\longrightarrow} * \rho^{\prime}\right\}
$$

would have been a perfectly fine co-inductive compliance. Note, though, that the client $\rho$ is by no means satisfied by the server.

Example 3.18. Note that according to our definition of compliance, the client need not ever perform $\checkmark$. For example, suppose $\alpha \bowtie \beta$ and consider the set

$$
\{(\mu x . \alpha . x, \mu y . \beta . y)\}
$$

It is a co-inductive compliance relation, and the client contract, $\mu x . \alpha . x$, does not perform $\checkmark$ at all.

Example 3.19. The fact that NIL can not be satisfied is formally expressed by the fact that

$$
\text { NIL } \Varangle \sigma
$$


for every contract $\sigma$. On the other hand $\mathbf{1}$ is always satisfied because for every $\sigma$

$$
1 \dashv \sigma .
$$

Suppose $\sigma$ is a contract which can not interact with the action $\alpha$; by this we mean that $\sigma \stackrel{\tau}{\longrightarrow} * \stackrel{\beta}{\longrightarrow}$ implies $\beta \bowtie \alpha$. Then

$$
1+\alpha . \rho \dashv \sigma
$$

for every $\rho$, because $\rho$ is guarded by an action that can never take place.

Referring to Example 3.13, it is routine work to check that the following relation is a co-inductive compliance.

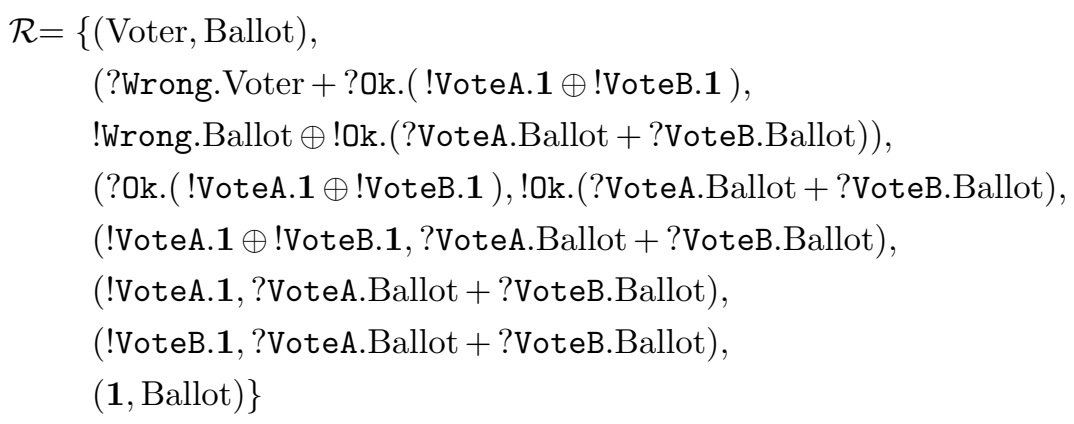

The compliance relation is determined purely by the client contract performing the success action $\checkmark$, therefore $\mathbf{1}$ complies with every contract $\sigma$; this means that a client whose contract is $\mathbf{1}$ is satisfied by any server. On the other hand a server with contract $\mathbf{1}$ is equivalent to a server with contract NIL. We prove this fact.

Proposition 3.20. For every contract $\rho, \rho \dashv 1$ if and only if $\rho \dashv$ NIL.

Proof. Suppose $\rho \dashv \mathbf{1}$; this means that there exists a co-inductive compliance $\mathcal{R}$ that contains $(\rho, \mathbf{1})$. Since $\mathbf{1}$ offers no interaction, the contract $\rho$ enjoys the two properties which follow,

(a) if $\rho \stackrel{\tau}{\lrcorner}$ then $\rho \stackrel{\checkmark}{\longrightarrow}$

(b) if $\rho \stackrel{\tau}{\longrightarrow} \rho^{\prime}$ then $\left(\rho^{\prime}, \mathbf{1}\right) \in \mathcal{R}$

Knowing (a) , it is straightforward to show that

$$
\mathcal{R}^{\prime} \triangleq\left\{\left(\rho^{\prime}, \mathrm{NIL}\right) \mid \rho \stackrel{\tau}{\longrightarrow}^{*} \rho^{\prime}, \rho \dashv \mathbf{1}\right\}
$$

is a co-inductive compliance.

A symmetrical argument can be used to show that also

$$
\mathcal{R}^{\prime} \triangleq\left\{\left(\rho^{\prime}, \mathbf{1}\right) \mid \rho \stackrel{\tau}{\longrightarrow}^{*} \rho^{\prime}, \rho \dashv \mathrm{NIL}\right\}
$$

is a co-inductive compliance. 
The following properties of the compliance relation will be useful later in the paper.

Lemma 3.21. Let $\rho, \sigma_{1}$, and $\sigma_{2}$ be contracts. The following hold:

(i) if $\rho \dashv \sigma_{1}, \rho \dashv \sigma_{2}$ then $\rho \dashv \sigma_{1} \oplus \sigma_{2}$

(ii) if $\rho_{1} \dashv \sigma, \rho_{2} \dashv \sigma$ then $\rho_{1} \oplus \rho_{2} \dashv \sigma$

Proof. As an example we outline the proof of (i). Let $\mathcal{R}$ be the relation defined by

$$
\mathcal{R}=\left\{(\rho, \sigma) \mid \rho \dashv \sigma \text { or } \sigma=\sigma_{1} \oplus \sigma_{2} \text { where } \rho \dashv \sigma_{1} \text { and } \rho \dashv \sigma_{2}\right\}
$$

It is straightforward to show that $\mathcal{R}$ is a compliance relation, from which the result follows.

Proposition 3.22. For all contracts $\rho, \sigma$, we have the following

(a) if $\rho \dashv \sigma$ then $\rho \dashv \operatorname{UNFOLD}(\sigma)$

(b) if $\rho \dashv \sigma$ then $\operatorname{UNFOLD}(\rho) \dashv \sigma$

Proof. Both follow in a straightforward manner from part (ii) of Lemma 3.2 and point (iii) of Definition 3.16 .

The converse is also true:

Proposition 3.23. For all contracts $\rho, \sigma$, we have the following

(a) if $\rho \dashv \operatorname{UNFOLD}(\sigma)$ then $\rho \dashv \sigma$

(b) if $\operatorname{UNFOLD}(\rho) \dashv \sigma$ then $\rho \dashv \sigma$

Proof. Let us look at the proof of (a). Let

$$
\mathcal{R}=\{(\rho, \sigma) \mid \rho \dashv \sigma \text { or } \rho \dashv \operatorname{UNFOLD}(\sigma)\}
$$

The result will follow if we can prove that $\mathcal{R}$ is a co-inductive compliance relation, as given in Definition 3.16

(i) Suppose $\rho \| \sigma \stackrel{\tau}{\hookrightarrow}$. If $\rho \dashv \sigma$ then by definition $\rho \stackrel{\checkmark}{\longrightarrow}$. Otherwise

$$
\rho \dashv \operatorname{UNFOLD}(\sigma)
$$

Note that $\sigma \stackrel{\tau}{\nrightarrow}$ and therefore by part (i) of Lemma 3.2 it follows that $\operatorname{UNFOLD}(\sigma)=\sigma$, which means, since now $\rho \dashv \sigma, \rho \stackrel{\checkmark}{\longrightarrow}$.

(ii) Suppose $\rho\left\|\sigma \stackrel{\tau}{\longrightarrow} \rho^{\prime}\right\| \sigma^{\prime}$. We have to show $\left(\rho^{\prime}, \sigma^{\prime}\right) \in \mathcal{R}$, which is obvious if $\rho \dashv \sigma$. On the other hand if $\rho \dashv \operatorname{UNFOLD}(\sigma)$ there are three cases, depending on the inference of the action $\rho\left\|\sigma \stackrel{\tau}{\longrightarrow} \rho^{\prime}\right\| \sigma^{\prime}$. If the action is due to a silent move of $\rho$, the result follows from point (iii) of Definition 3.16 . In the other cases the result will follow by an application of parts (ii), (iii) or (iv) of Lemma 3.2 and of point (iii) of Definition 3.16. 


\subsection{The server pre-order}

In this subsection we show how to compare servers in terms of their ability to satisfy clients; once more this is done in terms of their respective contracts.

Definition 3.24. [Server pre-order] Let $\llbracket \sigma \rrbracket_{\mathrm{SRV}}=\{\rho \mid \rho \dashv \sigma\}$. Then we write $\sigma \sqsubseteq_{\mathrm{SRV}} \sigma^{\prime}$ whenever $\llbracket \sigma \rrbracket_{\mathrm{SRV}} \subseteq \llbracket \sigma^{\prime} \rrbracket_{\mathrm{SRV}}$.

This provides us with a natural subsumption-like pre-order between serverside contracts. For if $\sigma \sqsubseteq_{\mathrm{SRV}} \sigma^{\prime}$ then we are assured every client satisfied by a server running the contract $\sigma$ is by definition also satisfied by a server running the contract $\sigma^{\prime}$.

One consequence of Proposition 3.22 and Proposition 3.23 is that

$$
\llbracket \operatorname{UNFOLD}(\sigma) \rrbracket_{\mathrm{SRV}}=\llbracket \sigma \rrbracket_{\mathrm{SRV}}
$$

and therefore when reasoning about the server pre-order we can work up to unfolding.

\subsubsection{Co-inductive characterisation}

Here we give a co-inductive characterisation of the server pre-order $\bigsqcup_{\mathrm{SRV}}$; this is based on a number of semantic properties of contracts, which we outline in the following lemmas.

Lemma 3.25. If $\sigma_{1} \sqsubseteq_{\mathrm{SRV}} \sigma_{2}$ and $\sigma_{2} \stackrel{\tau}{\longrightarrow} \sigma_{2}^{\prime}$ then $\sigma_{1} \sqsubseteq_{\mathrm{SRV}} \sigma_{2}^{\prime}$.

Proof. Suppose $\rho \dashv \sigma_{1}$, where $\sigma_{1} \sqsubseteq_{\text {SRv }} \sigma_{2}$ and $\sigma_{2} \stackrel{\tau}{\longrightarrow} \sigma_{2}^{\prime}$; we have to show $\rho \dashv \sigma_{2}^{\prime}$.

We know $\dashv$ is a co-inductive compliance relation, and also that $\rho \| \sigma_{2} \stackrel{\tau}{\longrightarrow}$ $\rho \| \sigma_{2}^{\prime}$. So by part (ii) of Definition 3.16 the required $\rho \dashv \sigma_{2}^{\prime}$ follows.

The next property involves the acceptance sets of contracts. For any $\mathrm{R} \subseteq$ Act let us write $\sigma \downarrow \mathrm{R}$ whenever $\sigma \stackrel{\tau}{\hookrightarrow}$ and $\mathrm{R}=\{\alpha \in$ Act $\mid \sigma \stackrel{\alpha}{\longrightarrow}\}$. These sets $\mathrm{R}, \mathrm{R}^{\prime}, \ldots$ are called initials [EF02] or ready sets [LP07, LP08]. If $\sigma \downarrow \mathrm{R}$ we say that $\sigma$ converges to $\mathrm{R}$; indeed, in our presentation only stuck states have ready sets.

Then let $\operatorname{ACC}(\sigma)=\left\{\mathrm{R} \mid \sigma \stackrel{\tau}{\longrightarrow} \sigma^{\prime}, \sigma^{\prime} \downarrow \mathrm{R}\right\}$; these are called the acceptance sets of $\sigma$.

Example 3.26. We give a small example about the definitions above. See also Figure 8 .

$$
\begin{array}{ll}
\sigma=\alpha . \sigma_{1} \oplus\left(\beta . \sigma_{2} \oplus \gamma \cdot \sigma_{3}\right) & \left.\sigma^{\prime}=\left(\alpha . \sigma_{1}^{\prime} \oplus \beta . \sigma_{2}\right) \oplus \gamma \cdot \sigma_{3}\right) \\
\operatorname{ACC}(\sigma)=\{\{\alpha\},\{\beta\},\{\gamma\}\} & \operatorname{ACC}(\sigma)=\{\{\alpha\},\{\beta\},\{\gamma\}\}
\end{array}
$$

Moreover, one sees easily that NIL $\downarrow \emptyset$ and $\mathbf{1} \downarrow \emptyset$, so

$$
\operatorname{ACC}(\mathrm{NIL})=\operatorname{ACC}(\mathbf{1})=\{\emptyset\}
$$



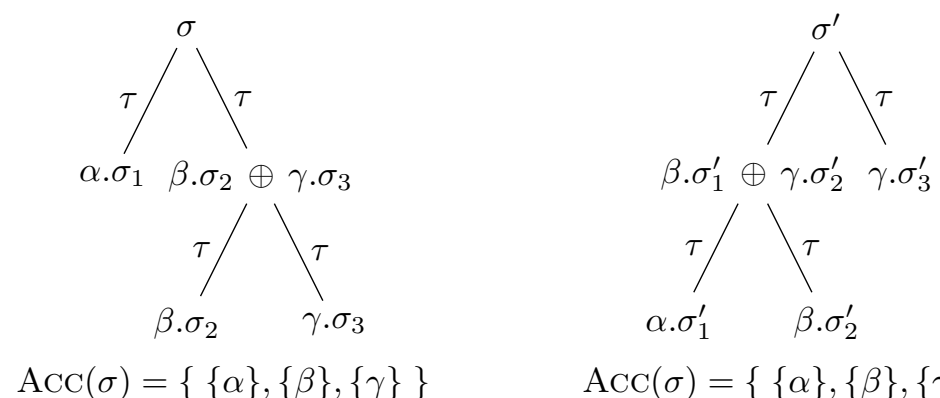

$\operatorname{ACC}(\sigma)=\{\{\alpha\},\{\beta\},\{\gamma\}\}$

$\operatorname{ACC}(\sigma)=\{\{\alpha\},\{\beta\},\{\gamma\}\}$

Figure 8: An example of acceptance sets.

Example 3.27. It is easy to show that whenever $\operatorname{UNFOLD}(\sigma)=1$ and $\sigma \downarrow \mathrm{R}$ then $\mathrm{R} \sqsubseteq \mathrm{R}^{\prime}$ for every $\mathrm{R}^{\prime}$. Consider the action $\checkmark$; it does not belong to Act, therefore by definition of $\sigma \downarrow$ R the action $\checkmark$ is not in R. But $\checkmark$ is the only visible action performed by $\sigma$, hence $\mathrm{R}=\emptyset$.

Proposition 3.28. Let $\sigma \in \mathcal{C}$. Then

(a) if $\sigma \downarrow \mathrm{R}$ then R is finite

(b) the set $\operatorname{Acc}(\sigma)$ is finite

(c) the set $\operatorname{Acc}(\sigma)$ is non-empty

Proof. Part (a) follows from the fact that external sums contain a finite amount of summands. Part (b) follows form Lemma 3.11. Part (c) follows from the fact that if $\sigma \downarrow$ then $\sigma$ has stuck derivatives.

Example 3.29. [Divergent terms and acceptance sets]

Here we show the acceptance set of a divergent term. Let $\sigma=\mu x . \alpha . x+x$. For every R we have $\sigma \Downarrow \mathrm{R}$ because $\sigma \stackrel{\tau}{\longrightarrow}$, and the same is true for the derivative $\alpha . \sigma+\sigma$, because it also performs a $\tau$ :

$$
\sigma \stackrel{\tau}{\longrightarrow} \alpha . \sigma+\sigma \stackrel{\tau}{\longrightarrow} \ldots
$$

We thus conclude that $\operatorname{Acc}(\sigma)=\emptyset$. Indeed, in the proof of part (iii) of the preceding proposition the crucial hypothesis is $\sigma \downarrow$.

Individual acceptance sets are compared by their ability to offer interactions. We write $\mathrm{R} \sqsubseteq \mathrm{S}$ whenever for every $\alpha_{\mathrm{R}} \in \mathrm{R}$ and every $\beta$ such that $\beta \bowtie \alpha_{\mathrm{R}}$ there exists some $\alpha_{\mathrm{S}} \in \mathrm{S}$ such that $\beta \bowtie \alpha_{\mathrm{S}}$ also. The precise meaning of this pre-order actually depends on the instantiation of the interaction relation $\bowtie$.

Example 3.30. Suppose Act is the set $\{a$ ?, $a ! \mid a \in A\}$ and $\bowtie_{i}$ is defined as in Example 3.15. Then one can check that $\mathrm{R} \sqsubseteq \mathrm{s}$ if and only if $R \subseteq S$. On the other hand with $\bowtie_{c}$ from the same example, defined over the set of actions $\{? \mathrm{~b}, ! \mathrm{b} \mid \mathrm{b} \in \mathrm{BT}\} \cup\{\mathrm{l} ?, \mathrm{l} ! \mid \mathrm{l} \in \mathbb{L}\}$, it turns out that $\mathrm{R} \sqsubseteq \mathrm{S}$ whenever

- $! \mathrm{l} \in \mathrm{R}$ implies $\mathrm{l} \in \mathrm{S}$ for every $\mathrm{l} \in \mathrm{L}$ 
- $? l \in$ ? implies $l \in \mathrm{S}$ for every $l \in \mathrm{L}$

- $? \mathrm{~b}_{\mathrm{R}} \in \mathrm{R}$ implies $? \mathrm{~b}_{\mathrm{S}} \in \mathrm{S}$ for some type $\mathrm{b}_{\mathrm{S}}$ such that $\mathrm{b}_{\mathrm{S}} \preccurlyeq_{\mathrm{g}} \mathrm{b}_{\mathrm{R}}$

- $! b_{R} \in R$ implies $! b_{S} \in \mathrm{s}$ for some type $b_{s}$ such that $b_{R} \preccurlyeq_{g} b_{S}$

Lemma 3.31. Suppose $\sigma_{1} \sqsubseteq_{\mathrm{SRV}} \sigma_{2}$ and $\sigma_{2} \downarrow \mathrm{R}$, then there is some $\mathrm{R}^{\prime} \in \operatorname{ACC}\left(\sigma_{1}\right)$ such that $\mathrm{R}^{\prime} \sqsubseteq \mathrm{R}$.

Proof. This proof proceeds by contradiction. To establish the contradiction we construct a contract $\rho$ such that

(i) $\rho \dashv \sigma_{1}$

(ii) $\rho \not \gamma \sigma_{2}$

Suppose there is no $\mathrm{R}^{\prime} \in \operatorname{ACC}\left(\sigma_{1}\right)$ such that $\mathrm{R}^{\prime} \sqsubseteq \mathrm{R}$. Thanks to part (C) of Proposition 3.28 this can not be because $\operatorname{ACC}\left(\sigma_{1}\right)$ is empty. Again by Proposition 3.28 we know $\operatorname{ACC}\left(\sigma_{1}\right)$ to be finite, so let $\mathrm{R}_{1}, \ldots \mathrm{R}_{n}$ be all the elements in $\operatorname{AcC}\left(\sigma_{1}\right)$. From the hypothesis there are $\alpha_{i} \in \mathrm{R}_{i}$ and $\beta_{i} \bowtie \alpha_{i}$ such that $\beta_{i} \bowtie \alpha$ whenever $\alpha \in \mathrm{R}^{\prime}$. Let the contract $\rho$ be defined as $\beta_{1} . \mathbf{1}+\ldots+\beta_{n} . \mathbf{1}$.

First notice that (ii) above is true: since $\sigma_{2} \downarrow \mathrm{R}, \rho\left\|\sigma_{2} \stackrel{\tau}{\longrightarrow}^{*} \rho\right\| \sigma_{2}^{\prime}$ such that $\rho \| \sigma_{2}^{\prime} \stackrel{\tau}{\dagger}$ and $\rho \stackrel{\checkmark}{\dagger}$. This means that $\left(\rho, \sigma_{2}\right)$ can not be contained in any co-inductive compliance relation.

To establish (i) above it is sufficient to prove that

$$
\mathcal{R}=\left\{\left(\rho, \sigma_{1}^{\prime}\right) \mid \sigma_{1} \stackrel{\tau}{\longrightarrow}^{*} \sigma_{1}^{\prime}\right\}
$$

is a co-inductive compliance relation, which is relatively straightforward.

The final property is even more complicated. For any action $\alpha$ let $\sigma$ AFTER $\alpha$ be the set

$$
\left\{\sigma^{\prime} \mid \sigma \stackrel{\tau}{\longrightarrow}^{*} \stackrel{\beta}{\longrightarrow} \stackrel{\tau}{\longrightarrow} \sigma^{\prime} \text {, where } \alpha \bowtie \beta\right\}
$$

Proposition 3.32. Let $\sigma$ be a contract, then for every $\alpha \in$ Acts the set $(\sigma$ AFTER $\alpha)$ is finite.

Proof. Because we assume $\bowtie$ to be finitary and for every contract $S(\sigma)$ and $F(\sigma)$ are finite (see Lemma 3.5 and Lemma 3.11).

Note that in general this set may be empty. When it is non-empty we use $\bigoplus(\sigma \operatorname{AFTER} \alpha)$ to denote the obvious contract, the internal sum of all its element:2

Lemma 3.33. Suppose $\sigma_{1} \sqsubseteq_{\mathrm{SRV}} \sigma_{2}$ and $\sigma_{2} \stackrel{\beta}{\longrightarrow} \sigma_{2}^{\prime}$. Then whenever $\alpha \bowtie \beta$,

(i) the set $\left(\sigma_{1} \operatorname{AFTER} \alpha\right)$ is non-empty

(ii) the contract $\bigoplus\left(\sigma_{1}\right.$ AFTER $\left.\alpha\right)$ is smaller than $\sigma_{2}^{\prime}$. Formally,

$$
\bigoplus\left(\sigma_{1} \operatorname{AFTER} \alpha\right) \sqsubseteq_{\mathrm{SRV}} \sigma_{2}^{\prime}
$$

\footnotetext{
${ }^{2}$ A concise account for the use of the general sum operation $\bigoplus$ is given in footnote 4 , Section 4
} 
Proof. To prove part (i) consider the contract $\rho=\mathbf{1}+\alpha$.NIL. Since $\rho \| \sigma_{2} \stackrel{\tau}{\longrightarrow}$ NIL $\| \sigma_{2}^{\prime}$ it follows that $\left(\rho, \sigma_{2}^{\prime}\right)$ can not be in any co-inductive compliance relation, hence $\rho \Varangle \sigma_{2}$. Therefore, from $\sigma_{1} \sqsubseteq_{\text {SRV }} \sigma_{2}$, we have that $\rho \Varangle \sigma_{1}$.

But because of the construction of $\rho$ this can only be the case if ( $\sigma_{1}$ AFTER $\alpha$ ) is non-empty. More specifically, if it was empty we could construct a simple coinductive compliance relation containing the pair $\left(\rho, \sigma_{1}\right)$.

Now consider part (ii). Suppose $\rho \dashv \bigoplus(\sigma$ AFTER $\alpha)$; we have to show $\rho \dashv \sigma_{2}^{\prime}$. To do so consider the contract $\rho^{\prime}=\mathbf{1}+\alpha . \rho$. Suppose we could establish

$$
\rho^{\prime} \dashv \sigma_{1}
$$

Because $\sigma_{1} \sqsubseteq_{\mathrm{SRV}} \sigma_{2}$ this would mean that $\rho^{\prime} \dashv \sigma_{2}$, from which the required $\rho \dashv \sigma_{2}^{\prime}$ follows, by part (ii) of Definition 3.16 .

It remains to prove (2) above. Let

$$
\mathcal{R}=\left\{\left(\rho, \sigma^{\prime}\right) \mid \rho \dashv \sigma^{\prime}, \sigma^{\prime} \in \mathcal{C}\right\} \cup\left\{\left(\rho^{\prime}, \sigma_{1}^{\prime}\right) \mid \sigma_{1} \stackrel{\tau}{\longrightarrow}^{*} \sigma_{1}^{\prime}\right\}
$$

Then, because $\rho \dashv \bigoplus(\sigma$ AFTER $\alpha)$, it is easy to establish that $\mathcal{R}$ is a co-inductive compliance relation.

We have now assembled all the required properties for our co-inductive characterisation of the server pre-order.

Definition 3.34. [Semantic sub-server relation] Let $\mathcal{F}_{\preccurlyeq_{\text {SRV }}}: \mathcal{P}\left(\mathcal{C}^{2}\right) \longrightarrow \mathcal{P}\left(\mathcal{C}^{2}\right)$ be the function defined so that $\left(\sigma_{1}, \sigma_{2}\right) \in \mathcal{F}_{\preccurlyeq_{\text {sRv }}}(\mathcal{R})$ if and only if the following conditions hold:

(i) if $\sigma_{2} \stackrel{\tau}{\longrightarrow} \sigma_{2}^{\prime}$ then $\left(\sigma_{1}, \sigma_{2}^{\prime}\right) \in \mathcal{R}$

(ii) for every $\mathrm{R} \in \operatorname{ACC}\left(\sigma_{2}\right), \sigma_{2} \downarrow \mathrm{R}$ implies $\mathrm{R}^{\prime} \sqsubseteq \mathrm{R}$ for some $\mathrm{R}^{\prime} \in \operatorname{ACC}\left(\sigma_{1}\right)$

(iii) if $\sigma_{2} \stackrel{\beta}{\longrightarrow} \sigma_{2}^{\prime}$ and $\alpha \bowtie \beta$ then $\left(\sigma_{1}\right.$ AFTER $\left.\alpha\right) \neq \emptyset$ and $\bigoplus\left(\sigma_{1}\right.$ AFTER $\left.\alpha\right) \mathcal{R} \quad \sigma_{2}^{\prime}$

If $\mathcal{R} \subseteq \mathcal{F}_{\preccurlyeq \text { srv }}(\mathcal{R})$ then we say that $\mathcal{R}$ is a co-inductive semantic sub-server relation. Let $\preccurlyeq_{\mathrm{sRV}}$ denote the greatest solution of the equation $X=\mathcal{F}_{\preccurlyeq_{\mathrm{sRv}}}(X)$. We call this solution the semantic sub-server relation. The relation $\preccurlyeq_{\mathrm{sRV}}$ is the greatest co-inductive semantic sub-server relation.

Proposition 3.35. If $\sigma_{1} \sqsubseteq_{\mathrm{SRV}} \sigma_{2}$ then $\sigma_{1} \preccurlyeq_{\mathrm{SRV}} \sigma_{2}$.

Proof. It is sufficient to prove that the relation $\sqsubseteq_{\mathrm{SRV}}$ is a semantic sub-server relation; this is straightforward in view of the last three lemmas.

We also have the converse.

Theorem 3.36. [Co-inductive characterisation]

The server pre-order is the greatest semantic sub-server relation.

Proof. We are required to prove that for all contracts $\sigma_{1}, \sigma_{2}$,

$$
\sigma_{1} \sqsubseteq_{\mathrm{SRV}} \sigma_{2} \text { if and only if } \sigma_{1} \preccurlyeq_{\mathrm{SRV}} \sigma_{2}
$$

Because of the previous proposition it is sufficient to prove that $\sigma_{1} \preccurlyeq_{\mathrm{SRV}} \sigma_{2}$ and $\rho \dashv \sigma_{1}$ implies $\rho \dashv \sigma_{2}$. This will follow if we can show that the relation

$$
\mathcal{R}=\left\{(\rho, \sigma) \mid \rho \dashv \sigma_{1} \text { for some } \sigma_{1} \text { such that } \sigma_{1} \preccurlyeq_{\mathrm{SRV}} \sigma\right\}
$$

is a co-inductive compliance relation.

Suppose $(\rho, \sigma) \in \mathcal{R}$. By Definition 3.16 we are required to show that 


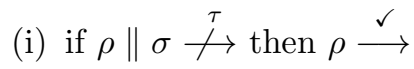

(ii) if $\rho\left\|\sigma \stackrel{\tau}{\longrightarrow} \rho^{\prime}\right\| \sigma^{\prime}$ then $\left(\rho^{\prime}, \sigma^{\prime}\right) \in \mathcal{R}$

By the definition of $\mathcal{R}$ we know that there is some contract $\sigma_{1}$ such that $\sigma_{1} \preccurlyeq$ sRv $\sigma$ and $\rho \dashv \sigma_{1}$.

We prove the first point, (i). If $\rho \| \sigma \stackrel{\tau}{\dagger}$ then $\rho \stackrel{\tau}{\dagger}, \sigma \stackrel{\tau}{\nrightarrow}$; in addition, the two contracts can not interact, that is $\rho \stackrel{\alpha}{\longrightarrow}$ and $\sigma \stackrel{\beta}{\longrightarrow}$ implies $\alpha \bowtie \beta$. Since $\rho$ and $\sigma$ are stable both $\operatorname{ACC}(\rho)$ and $\operatorname{ACC}(\sigma)$ contain exactly one set each, say R and $\mathrm{s}$ respectively. Then rephrasing the above remark we know

$$
\alpha \in \mathrm{R}, \beta \in \mathrm{S} \text { implies } \alpha \bowtie \beta
$$

Since $\sigma_{1} \preccurlyeq$ SRv $\sigma$, by part (ii) of Definition $3.34 \sigma_{1} \stackrel{\tau}{\longrightarrow}^{*} \sigma_{1}^{\prime}$ for some $\sigma_{1}^{\prime}$ such that $\sigma_{1}^{\prime} \downarrow \mathrm{s}^{\prime}$ and $\mathrm{s}^{\prime} \sqsubseteq \mathrm{s}$. One can use (2) above to show that this means

$$
\alpha \in \mathrm{R}, \beta \in \mathrm{s}^{\prime} \text { implies } \alpha \bowtie \beta
$$

Also, since $\rho \dashv \sigma_{1}$ and $\sigma_{1} \stackrel{\tau}{\longrightarrow}^{*} \sigma_{1}^{\prime}$, part (ii) of Definition 3.16 implies $\rho \dashv \sigma_{1}^{\prime}$. But $\rho \| \sigma_{1}^{\prime} \stackrel{\tau}{\dagger}$ and therefore we have the required $\rho \stackrel{\checkmark}{\longrightarrow}$.

To prove point (ii) above, we have to show that if $\rho\left\|\sigma \stackrel{\tau}{\longrightarrow} \rho^{\prime}\right\| \sigma^{\prime}$ there exists a $\hat{\sigma}$ such that

$$
\rho^{\prime} \dashv \hat{\sigma} \text { and } \hat{\sigma} \preccurlyeq_{\mathrm{SRV}} \sigma^{\prime}
$$

We proceed by case analysis on the rule used to infer $\rho\left\|\sigma \stackrel{\tau}{\longrightarrow} \rho^{\prime}\right\| \sigma^{\prime}$. There are three possibilities: first suppose the inference rule [P-SiL-L] from Figure 7 is used; that is $\rho \stackrel{\tau}{\longrightarrow} \rho^{\prime}$ and $\sigma^{\prime}=\sigma$. In this case the required $\hat{\sigma}$ is $\sigma_{1}$; the definition of $\mathcal{R}$ gives $\sigma_{1} \preccurlyeq_{\mathrm{SRV}} \sigma$ and point (ii) of the definition of compliance, Definition 3.16, gives $\rho^{\prime} \dashv \sigma_{1}$.

The case when the rule [P-SIL-R] is used is similar; choosing the required $\hat{\sigma}$ to be $\sigma_{1}$ again is justified by point (i) of Definition 3.34

Finally suppose [P-SYNCH] is employed. Now we know that

$$
\rho \stackrel{\delta}{\longrightarrow} \rho^{\prime}, \quad \sigma \stackrel{\beta}{\longrightarrow} \sigma^{\prime}, \quad \delta \bowtie \beta .
$$

In this case we show that the required $\hat{\sigma}$ is $\bigoplus\left(\sigma_{1}\right.$ AFTER $\left.\delta\right)$. Part (iii) of Definition 3.34 implies that $\bigoplus\left(\sigma_{1}\right.$ AFTER $\left.\delta\right) \preccurlyeq{ }_{\mathrm{SRV}} \sigma^{\prime}$ and thus it suffices to show that $\rho^{\prime} \dashv \bigoplus\left(\sigma_{1}\right.$ AFTER $\left.\delta\right)$.

The set $\sigma_{1}$ AFTER $\delta$ is finite and therefore by Lemma 3.21 it is sufficient to prove $\rho^{\prime} \dashv \sigma^{\prime \prime}$ for every $\sigma^{\prime \prime} \in\left(\sigma_{1} \operatorname{AFTER} \delta\right)$.

For such a $\sigma^{\prime \prime}$ we can derive the transition

$$
\rho\left\|\sigma_{1} \stackrel{\tau}{\longrightarrow} \rho^{\prime}\right\| \sigma^{\prime \prime}
$$

where one of the reductions is due to the interaction through $\delta$. From part (ii) of Definition 3.16 it follows that $\rho \dashv \sigma^{\prime \prime}$. 


\subsection{Must testing}

The compliance relation between contracts, Definition 3.16, has much in common with the idea of testing from [NH84]. Here we explain the relationship. We recall the definition of MUST testing, and explain how it differs from the compliance relation. Despite this difference we then go on to show that the testing pre-order it induces on contracts actually coincides with the server pre-order.

For every contract $\rho$ and $\sigma$ a sequence of reductions

$$
\rho\left\|\sigma \stackrel{\tau}{\longrightarrow} \rho_{1}\right\| \sigma_{1} \stackrel{\tau}{\longrightarrow} \rho_{2} \| \sigma_{2} \longrightarrow \ldots
$$

is called a computation of $\rho \| \sigma$ and each derivative $\rho_{i} \| \sigma_{i}$ is a state of the computation. Intuitively viewing $\rho$ as a test, we say that the state $\rho_{i} \| \sigma_{i}$ is successful if $\rho_{i} \stackrel{\checkmark}{\longrightarrow}$. Then, a computation is successful it it contains a successful state.

Example 3.37. For every $\sigma$ all the computations of

$$
1+? 1_{1} \text {.NIL } \| \sigma
$$

are successful because in the first state $1 \stackrel{\checkmark}{\longrightarrow}$. On the other hand, suppose a contract $\rho$ does not perform $\checkmark$ and neither do its derivatives. Then no computation of $\rho \| \sigma$ is successful.

A computation is maximal if either

(i) it is infinite, or

(ii) it is finite and the last state is stuck, that is has the form $\rho_{k} \| \sigma_{k}$ where

$$
\rho_{k} \| \sigma_{k} \stackrel{\tau}{\perp}
$$

Definition 3.38. [Must testing]

For all contracts $\rho, \sigma$ we write $\sigma \operatorname{MUST} \rho$ if all the maximal computations of $\rho \| \sigma$ are successful.

The notion of a client contract complying with a server contract differs in two ways from that of a server contract passing a client contract viewed as a test.

Example 3.39. One difference between MUST and $\dashv$ is what happens after a contract has passed a test, that is the test has reached a success full state; the subsequent computation is disregarded by MUST, whereas the compliance relation has to hold for all the states in a computation.

As an example consider $\sigma=$ ?Real.NIL and $\rho=1+$ !Int.NIL. Clearly $\rho \stackrel{\checkmark}{\longrightarrow}$, therefore $\sigma$ MUST $\rho$ because each maximal computation of $\rho \| \sigma$ begins in a successful state. However $\rho \nrightarrow \sigma$ because $\rho \| \sigma \stackrel{\tau}{\longrightarrow}$ NIL $\|$ NIL.

Example 3.40. The second difference is that the compliance relation does not require the testing contract to ever report success, provided that the communication between the contracts can continue indefinitely. As an example consider the following contracts

$$
\sigma=\mu x . \text { !Bool. } x, \quad \rho=\mu y . \text { ?Rnd. } x+\text { !Int.1 }
$$


Plainly, one sees that $\rho \stackrel{\checkmark}{\sim}$, and, therefore, $\rho \| \sigma$ is not a successful state. The only computation of $\rho \| \sigma$ is the infinite loop $\rho\|\sigma \stackrel{\tau}{\longrightarrow} \rho\| \sigma$, and therefore $\rho \dashv \sigma$ holds; on the other hand $\sigma$ MUST $\rho$ is false. Example 3.18 contains an even simpler instance of the difference between the relation $\dashv$ and the relation MUST.

The MUST relation can be used to define a well known pre-order:

Definition 3.41 (Must pre-order [NH84]). Let $\sigma_{1}, \sigma_{2}$ be contracts. We write $\sigma_{1} \sqsubseteq_{\text {MUST }} \sigma_{2}$ if and only if for every $\rho$ if $\sigma_{1} \operatorname{MUST} \rho$ then $\sigma_{2}$ MUST $\rho$.

Notwithstanding the differences between testing and compliance discussed above, it turns out that the server pre-order $\sqsubseteq_{\mathrm{SRV}}$ and the MUST pre-order $\sqsubseteq_{\text {MUST }}$ coincide (Corollary 3.49).

First, in a series of lemmas, we show that $\sqsubseteq_{\text {мUsт }}$ satisfies the three defining properties of the semantic sub-server relation (Definition 3.34).

Lemma 3.42. Let $\sigma_{1} \sqsubseteq_{\text {мust }} \sigma_{2}$. If $\sigma_{2} \stackrel{\tau}{\longrightarrow} \sigma_{2}^{\prime}$ then $\sigma_{1} \sqsubseteq_{\text {мUsт }} \sigma_{2}^{\prime}$.

Proof. Take a contract $\rho$ such that $\sigma_{1}$ MUST $\rho$ and a maximal computation $C$ performed by $\sigma_{2}^{\prime} \| \rho$. It is easy to see that a maximal computation from $\sigma_{2} \| \rho$ can be obtained by prefixing $C$ with the move $\sigma_{2}\left\|\rho \stackrel{\tau}{\longrightarrow} \sigma_{2}^{\prime}\right\| \rho$.

Since $\sigma_{1} \sqsubseteq_{\text {мusт }} \sigma_{2}$ it follows that this extended computation must be successful. However this implies that $C$ itself is successful since $\rho$ does not change during the initial extending move.

Lemma 3.43. Let $\sigma_{1} \sqsubseteq_{\text {мUsт }} \sigma_{2}$ and $\sigma_{2} \downarrow$ R. There exists a $\mathrm{R}^{\prime} \in \operatorname{ACC}\left(\sigma_{1}\right)$ such that $\mathrm{R}^{\prime} \sqsubseteq \mathrm{R}$.

Proof. The proof is similar to that of Lemma 3.31, and proceeds by contradiction. Let $\mathrm{R}_{1}, \ldots, \mathrm{R}_{n}, n \geq 1$ be the elements of $\operatorname{ACC}\left(\sigma_{1}\right)$ and suppose that $\mathrm{R}_{i} \not E \mathrm{R}$. This means that for every $\mathrm{R}_{i}$ there is $\alpha_{i} \in \mathrm{R}_{i}$ and a $\beta_{i}$ such that $\beta_{i} \bowtie \alpha_{i}$ and $\beta_{i} \not \alpha$ whenever $\alpha \in \mathrm{R}$.

Let $\rho$ be the contract $\beta_{1} .1+\ldots+\beta_{n} . \mathbf{1}$. The contradiction is established by showing that

(i) $\sigma_{1} \operatorname{MUST} \rho$ while

(ii) $\sigma_{2} \operatorname{MUST} \rho$ is false

Both of which we leave to the reader. Intuitively (ii) follows because $\sigma_{2} \downarrow \mathrm{R}$, while (i) is a consequence of the fact that if $\sigma_{1} \stackrel{\tau}{\longrightarrow} \sigma^{\prime} \stackrel{\tau}{\perp}$ then $\sigma^{\prime} \downarrow \mathrm{R}_{i}$ for some $1 \leq i \leq n$.

Lemma 3.44. Let $\sigma_{1} \sqsubseteq_{\text {мust }} \sigma_{2}$ and $\sigma_{2} \stackrel{\beta}{\longrightarrow} \sigma_{2}^{\prime}$. Whenever $\alpha \bowtie \beta$

(i) the set $\left(\sigma_{1}\right.$ AFTER $\left.\alpha\right)$ is not empty

(ii) the contract $\bigoplus\left(\sigma_{1}\right.$ AFTER $\left.\alpha\right)$ is smaller than $\sigma_{2}^{\prime}$. Formally,

$$
\bigoplus\left(\sigma_{1} \text { AFTER } \alpha\right) \sqsubseteq_{\text {мUSт }} \sigma_{2}^{\prime}
$$


Proof. The proof of (i) is analogous to that of (i) in Lemma 3.33 but the contract to be used in this case is $\rho=(\mathbf{1} \oplus \mathbf{1})+\alpha$.NIL.

We prove point (ii) by contradiction. Suppose there is a contract $\rho^{\prime}$ such that $\bigoplus\left(\sigma_{1} \operatorname{AFTER} \alpha\right)$ MUST $\rho^{\prime}$ while $\sigma_{2}^{\prime}$ MUST $\rho^{\prime}$ is false. Now consider the contract $\rho=\alpha . \rho^{\prime} \oplus \mathbf{1}$. Clearly $\sigma_{2} \operatorname{MUST} \rho$ is false while $\sigma_{1}$ MUST $\rho$ is true. This contradicts the hypothesis $\sigma_{1} \sqsubseteq_{\text {Must }} \sigma_{2}$.

Proposition 3.45. If $\sigma_{1} \sqsubseteq_{\mathrm{MUST}} \sigma_{2}$ then $\sigma_{1} \preccurlyeq_{\mathrm{SRV}} \sigma_{2}$.

Proof. The previous three lemmas show that $\sqsubseteq_{\text {MUsT }}$ is a semantic sub-server relation, from which the result follows.

To establish the converse of this result we need to develop some additional notation. The first is a generalisation of the relation $\sigma$ AFTER $\alpha$ to $\sigma$ AFTER $u$ where $u$ is a non-empty sequence of actions from $A c t^{\star}$. This is defined by induction on the length of $u$, with the inductive case being

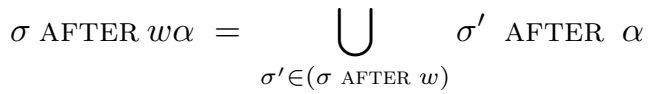

Example 3.46. Let $\sigma=! t_{1} \cdot\left(? t_{2} \cdot \sigma_{1}+? t_{3} \cdot \sigma_{2}\right)+! t_{1}$. NIL and $? t_{3} \bowtie ! t_{2}$. Then

$$
\begin{aligned}
\sigma \text { AFTER } ? \mathrm{t}_{1} ! \mathrm{t}_{2} & =\bigcup_{\sigma^{\prime} \in\left(\sigma \text { AFTER } ? \mathrm{t}_{1}\right)} \sigma^{\prime} \text { AFTER } ! \mathrm{t}_{2} \\
& =\bigcup_{\sigma^{\prime} \in\left\{\mathrm{NIL}, ? \mathrm{t}_{2}, \sigma_{1}+? \mathrm{t}_{3}, \sigma_{2}\right\}} \sigma^{\prime} \text { AFTER } ! \mathrm{t}_{2} \\
& =\left\{\sigma_{1}, \sigma_{2}\right\} .
\end{aligned}
$$

Next we generalise the interaction relation $\alpha \bowtie \beta$ to non-empty sequences, $u \bowtie w$ in the obvious manner; note that this implies that $u$ and $w$ have the same length. Finally we need the notion of contracts performing sequence of actions. For $u \in A c t^{\star}$ let $\sigma \stackrel{u}{\Longrightarrow} \sigma^{\prime}$ be the least relation which satisfies

(a) $\sigma \stackrel{\varepsilon}{\Longrightarrow} \sigma$ for every contract $\sigma$

(b) $\sigma \stackrel{u}{\Longrightarrow} \sigma_{1}, \sigma_{1} \stackrel{a}{\longrightarrow} \sigma^{\prime}$, where $a \in$ Act, implies $\sigma \stackrel{u . a}{\Longrightarrow} \sigma^{\prime}$

(c) $\sigma \stackrel{u}{\Longrightarrow} \sigma_{1}, \sigma_{1} \stackrel{\tau}{\longrightarrow} \sigma^{\prime}$ implies $\sigma \stackrel{u}{\Longrightarrow} \sigma^{\prime}$

We have the obvious generalisation of condition (iii) in Definition 3.34.

Lemma 3.47. Suppose $\sigma_{1} \mathcal{R} \sigma_{2}$ for some semantic sub-server relation $\mathcal{R}$ and $\sigma_{2} \stackrel{u}{\Longrightarrow} \sigma_{2}^{\prime}$ for some non-empty $u \in A c t^{\star}$. Then $v \bowtie u$ implies that

(i) the set $\left(\sigma_{1} \operatorname{AFTER} v\right)$ is not empty

(ii) the contract $\bigoplus\left(\sigma_{1}\right.$ AFTER $\left.v\right)$ is related by $\mathcal{R}$ to $\sigma_{2}^{\prime}$. Formally,

$$
\bigoplus\left(\sigma_{1} \operatorname{AFTER} v\right) \mathcal{R} \sigma_{2}^{\prime}
$$

Proof. By induction on the non-empty size of $u$; the base case follows from part (iii) of Definition 3.34 
Theorem 3.48. [Co-inductive characterisation]

The must pre-order is the greatest semantic sub-server relation.

Proof. We have to prove that for all contracts $\sigma_{1}, \sigma_{2}$

$$
\sigma_{1} \preccurlyeq_{\mathrm{SRV}} \sigma_{2} \text { if and only if } \sigma_{1} \sqsubseteq_{\text {MUST }} \sigma_{2} .
$$

Because of Proposition 3.45 it is sufficient to prove $\sigma_{1} \preccurlyeq_{\mathrm{SRV}} \sigma_{2}$ implies $\sigma_{1} \sqsubseteq_{\text {MUST }}$ $\sigma_{2}$. So, suppose $\sigma_{1} \preccurlyeq_{\text {SRV }} \sigma_{2}$ and $\sigma_{1}$ MUST $\rho$; we must prove $\sigma_{2}$ MUST $\rho$.

Consider a maximal computation of $\sigma_{2} \| \rho$

$$
\sigma_{2}\left\|\rho \stackrel{\tau}{\longrightarrow} \sigma_{2}^{1}\right\| \rho_{1} \stackrel{\tau}{\longrightarrow} \ldots
$$

We first examine the case when this is finite, with terminal state $\sigma_{2}^{k} \| \rho_{k}$. Intuitively this finite computation can be unzipped to give the contributions from the individual components $\sigma_{2}$ and $\rho$ :

$$
\sigma_{2} \stackrel{u}{\Longrightarrow} \sigma_{2}^{k} \quad \rho \stackrel{v}{\Longrightarrow} \rho_{k} \quad \text { where } v \bowtie u
$$

We are required to show that one of the derivatives of $\rho$ in $\rho \stackrel{v}{\Longrightarrow} \rho_{k}$ is successful. To this aim we will exhibit a suitable computation of $\sigma_{1} \| \rho$; in particular we will show that there exists a $\sigma_{1}^{\prime}$ such that

(a) the composition $\sigma_{1}^{\prime} \| \rho_{k}$ is stuck

(b) the computation $\sigma_{1}\left\|\rho \stackrel{\tau}{\longrightarrow}^{*} \sigma_{1}^{\prime}\right\| \rho_{k}$ exists

(c) the derivatives of $\rho$ in the computation of point (a) are contained in the computation $\sigma_{2}\left\|\rho \stackrel{\tau}{\longrightarrow}^{*} \sigma_{2}^{k}\right\| \rho_{k}$

These three points are enough to prove that in $\rho \stackrel{v}{\Longrightarrow}$ there exists a successful derivative: thanks to (a), the computation in (b) is a maximal computation of $\sigma_{1} \| \rho$; the assumption $\sigma_{1} \sqsubseteq_{\text {MUST }} \rho$ implies that in that computation there is a successful derivative $\hat{\rho}$, and point (c) ensures that $\hat{\rho}$ is contained in $\rho \stackrel{v}{\Longrightarrow}$.

We prove one by one the points above.

(a) Here we show that, for a suitable $\sigma_{1}^{\prime}$, the composition $\sigma_{1}^{\prime} \| \rho_{k}$ is stuck.

By assumption the state $\sigma_{2}^{k} \| \rho_{k}$ is terminal; this implies that

(1) both $\sigma_{2}^{k}$ and $\rho_{k}$ are stuck. A consequence is that their acceptance sets are singleton; say $\operatorname{ACC}\left(\sigma_{2}^{k}\right)=\{\mathrm{R}\}$ and $\operatorname{ACC}\left(\rho_{k}\right)=\{\mathrm{s}\}$

(2) the contracts $\sigma_{2}^{k}$ and $\rho_{k}$ can not interact. Formally

$$
\alpha \in \mathrm{R} \text { implies } \beta \bowtie \alpha \text { for every } \beta \in \mathrm{s} .
$$

Consider now the contract $\bigoplus\left(\sigma_{1}\right.$ AFTER $\left.v\right)$; Part (i) Lemma 3.47 implies that the set $\left(\sigma_{1}\right.$ AFTER $\left.v\right)$ is not empty and part (ii) of the same lemma implies that $\bigoplus\left(\sigma_{1}\right.$ AFTER $\left.v\right) \preccurlyeq_{\text {SRV }} \sigma_{2}^{k}$.

Part (ii) of Definition 3.34 and (2) above imply that there exists $\mathrm{R}^{\prime} \in$ $\operatorname{ACC}\left(\bigoplus\left(\sigma_{1}\right.\right.$ AFTER $\left.\left.v\right)\right)$ such that

$$
\alpha \in \mathrm{R}^{\prime} \text { implies } \beta \bowtie \alpha \text { for every } \beta \in \mathrm{s}^{\prime} .
$$


From the definition of acceptance set now follows that $\bigoplus\left(\sigma_{1}\right.$ AFTER $\left.v\right) \stackrel{\tau}{\longrightarrow}{ }^{*} \sigma_{1}^{\prime}$ for some $\sigma_{1}^{\prime}$ such that $\sigma_{1}^{\prime} \downarrow \mathrm{R}^{\prime}$. The latter fact means that $\sigma_{1}^{\prime} \stackrel{\tau}{\longrightarrow}$ and 4 above means that $\sigma_{1}^{\prime}$ and $\rho_{k}$ can not interact; Since (1) above proves that $\rho_{k}$ is stuck we have shown that $\sigma_{1}^{\prime} \| \rho_{k}$ is stuck.

(b) We are required to exhibit the computation $\sigma_{1}\left\|\rho \stackrel{\tau}{\longrightarrow}{ }^{*} \sigma_{1}^{\prime}\right\| \rho_{k}$.

Since $\bigoplus\left(\sigma_{1}\right.$ AFTER $\left.v\right) \stackrel{\tau}{\longrightarrow}{ }^{*} \sigma_{1}^{\prime}$ there exists a $\sigma_{1}^{\prime \prime} \in\left(\sigma_{1}\right.$ AFTER $\left.v\right)$ such that $\sigma_{1}^{\prime \prime} \stackrel{\tau}{\longrightarrow}{ }^{*} \sigma_{1}^{\prime}$. From the definition of $\left(\sigma_{1}\right.$ AFTER $\left.v\right)$ it follows that $\sigma_{1} \stackrel{w}{\Longrightarrow} \sigma_{1}^{\prime \prime}$ for some such that $w \bowtie v$, and this implies that $\sigma_{1} \stackrel{w}{\Longrightarrow} \sigma_{1}^{\prime}$. Zipping this action sequence together with $\rho \stackrel{v}{\Longrightarrow} \rho_{k}$ we obtain the computation

$$
\sigma_{1}\left\|\rho \stackrel{\tau}{\longrightarrow} \sigma_{1}^{\prime}\right\| \rho_{k} \stackrel{\tau}{\longrightarrow}
$$

We remark that the computation above is finite and can not be extended, hence it is maximal.

(c) The derivatives of $\rho$ in the computation

$$
\sigma_{1}\left\|\rho \stackrel{\tau}{\longrightarrow} \sigma_{1}^{\prime}\right\| \rho_{k}
$$

are contained in the computation $\sigma_{2}\left\|\rho \stackrel{\tau}{\longrightarrow}{ }^{*} \sigma_{2}^{k}\right\| \rho_{k}$ because the former computation has been obtained by zipping $\rho \stackrel{v}{\Longrightarrow} \rho_{k}$ with a computation made by $\sigma_{1}$.

Now suppose that the maximal computation (3) above is infinite. Then the result of unzipping gives infinite traces $u, v$ such that

$$
\sigma_{2} \stackrel{u}{\Longrightarrow} \rho \stackrel{v}{\Longrightarrow}
$$

Let us denote the finite prefixes of these traces of length $k$ by $u(k), v(k)$ respectively. By Lemma 3.47 we know that $\sigma_{1}$ AFTER $v(k)$ is non-empty, for every $k \geq 0$. This means that the LTS generated by $\sigma_{1}$ is infinite.

Now consider the sub-LTS consisting of all nodes $\sigma$ which can be reached from $\sigma_{1}$ using a weak move $\sigma_{1} \stackrel{w(k)}{\Longrightarrow} \sigma^{\prime}$ where $w(k)$ is some trace satisfying $w(k) \bowtie v(k)$. This sub-LTS is therefore infinite. It is also finite-branching and so by König's lemma it has an infinite path. By following this path from the root we get $\sigma_{1} \stackrel{w}{\Longrightarrow}$ such that $w(k) \bowtie v(k)$, for every $k \geq 0$.

This infinite computation can now be zipped with $\rho \stackrel{v}{\Longrightarrow}$ to obtain an infinite computation from $\sigma_{1} \| \rho$. Since $\sigma_{1} \operatorname{MUST} \rho$ it follows that there is some $\sigma_{2}^{k} \|$ $\rho_{k}$ in the maximal computation (3) above which is successful, and therefore $\sigma_{2} \operatorname{MUST} \rho$.

Corollary 3.49. The server pre-order equals the must pre-order. Formally

$$
\sqsubseteq_{\mathrm{SRV}}=\sqsubseteq_{\mathrm{MUST}}
$$

Proof. It is a consequence of Theorem 3.36 and Theorem 3.48 


$$
\begin{aligned}
\sigma, \rho::= & \\
& \mathbf{1} \\
& \sum_{i \in I} ? 1_{i} . \sigma \\
& \bigoplus_{i \in I} ! 1_{i} . \sigma \\
& \text { t. } \sigma \\
& \text { t. } \sigma \\
& x \\
& \mu x . \sigma
\end{aligned}
$$

\author{
Session Contracts \\ success \\ external choice, I finite, non-empty \\ internal choice, I finite, non-empty \\ input \\ output \\ contract variable \\ recursion
}

We impose the additional proviso that in a term the $1_{i}$ 's are pair-wise different.

Figure 9: Session contract grammar.

\section{Session Contracts}

Here we specialise the contract language, to a sub-language which will be the target of our interpretation of the session types from Section 2 This is the topic of the first sub-section. We then go on to re-examine the server pre-order as it applies to this sub-language; in particular we show that it can also be characterised co-inductively, this time using purely syntactical criteria. In the final section we give a similar co-inductive characterisation to a related subclient pre-order.

\subsection{Session contracts}

The syntax for the language $L_{\mathcal{S C}}$ is given in Figure 9. We work relative to a structural equivalence, generated by the following identities ${ }^{3}$

$$
\begin{array}{ll}
\sigma \oplus \rho=\rho \oplus \sigma & \sigma \oplus\left(\sigma^{\prime} \oplus \sigma^{\prime \prime}\right)=\left(\sigma \oplus \sigma^{\prime}\right) \oplus \sigma^{\prime \prime} \\
\sigma+\rho=\rho+\sigma & \sigma+\left(\sigma^{\prime}+\sigma^{\prime \prime}\right)=\left(\sigma+\sigma^{\prime}\right)+\sigma^{\prime \prime}
\end{array}
$$

This justifies the use of the general summation constructs for internal and external choices, which emphasises the intended restrictions in the language. We use $\mathcal{S C}$ to denote the set of terms $\sigma$ of $L_{\mathcal{S C}}$ such that $\sigma \downarrow$. We refer to these terms as session contracts. Note that $\mathcal{S C}$ is a subset of the more general language of contracts $\mathcal{C}$, but

- external choices are restricted to inputs on labels

- internal choices are restricted to outputs on labels

Note also that NIL is not a session contract. Instead we have chosen $\mathbf{1}$ to be the base contract, for reasons which will become apparent. Moreover, we already reasoned that a server contract $\mathbf{1}$ has the same behaviour as NIL (Proposition 3.20 .

Session contracts, due to the their restrictive syntax, enjoy some properties which we will use in the next sub-sections, and which we prove now.

\footnotetext{
${ }^{3}$ Formally the use of these equalities is justified by the relationship between $\dashv$ and the weak bisimulation equivalence Mil99].
} 
Lemma 4.1. Let $\sigma$ be a session contract. Then

(i) $\sigma \stackrel{\checkmark}{\longrightarrow}$ if and only if $\sigma=\mathbf{1}$

(ii) $\sigma \stackrel{\tau}{\longrightarrow} * \stackrel{\checkmark}{\longrightarrow}$ if and only if $\operatorname{UNFOLD}(\sigma)=\mathbf{1}$

(iii) $\sigma \stackrel{\alpha}{\longrightarrow}$ if and only if $\sigma \neq \mathbf{1}$

Proof. Part (i) follows from the restrictive syntax of session contract. The proof of part (ii) requires two arguments. The if side, $\operatorname{UnFOLD}(\sigma)=\mathbf{1}$ implies $\sigma \stackrel{\tau}{\longrightarrow} \stackrel{\checkmark}{\longrightarrow}$, is justified by part (ii) of Lemma 3.2 . The only if side, $\sigma \stackrel{\tau}{\longrightarrow}{ }^{*} \stackrel{\checkmark}{\longrightarrow}$ implies $\operatorname{UNFOLD}(\sigma)=\mathbf{1}$, can be proven by induction on the length of the sequence $\stackrel{\tau}{\longrightarrow}$; the base case being part (i) of this lemma. Part (iii) can be proven by structural induction.

\subsection{The server pre-order}

Definition 3.24 applies equally well to session contracts, but it is inappropriate as it compares session contracts from the point of view of satisfying clients who may use the more general contracts from Section 3 . Instead let us restrict attention to clients who also only run the more restricted session contracts.

Definition 4.2. [Restricted server pre-order]

For $\sigma_{1}, \sigma_{2} \in \mathcal{S C}$ let $\sigma_{1} \sqsubseteq_{\mathrm{SRV}}^{\mathcal{S C}} \sigma_{2}$ whenever $\rho \dashv \sigma_{1}$ implies $\rho \dashv \sigma_{2}$ for every $\rho$ in $\mathcal{S C}$.

This relation is more generous than $\sqsubseteq_{\mathrm{SRV}}$ in that it allows implementation refinement Pad10 to happen, as the following example shows.

\section{Example 4.3.}

$$
? 1_{1} .1 \sqsubseteq_{\mathrm{SRV}}^{\mathcal{S C}} ? 1_{2} .1+? 1_{1} \cdot 1
$$

If a session contract $\rho$ can interact with $? 1_{1} \cdot 1$ then, modulo unfolding, it has to be defined by an internal sum. Moreover this sum can only contain one summand and therefore $\rho$ complies also with $? \mathfrak{l}_{2} \cdot \mathbf{1}+? \mathfrak{1}_{1} \cdot \mathbf{1}$.

Consider now the more general contract $\rho^{\prime}=! 1_{1} \cdot 1+! 1_{2}$.NIL. Then one can check that $\rho^{\prime} \dashv ? 1_{1} .1$ whereas $\rho^{\prime}-\nmid ? 1_{2} \cdot 1+? 1_{1} .1$. It therefore follows that

$$
? 1_{1} \cdot 1 \not \mathbb{S R V}_{\mathrm{SRV}} ? 1_{2} \cdot 1+? 1_{1} \cdot 1
$$

Example 4.4. [e-vote, ballot refinement]

We give a more concrete instance of the previous example. Recall Example 3.13 and consider the session contract

$$
\begin{aligned}
& \text { BallotB }=\mu x . \text { ?Login. }(\text { !Wrong. } 1 \oplus \\
&\text { !Ok. }(\text { ?VoteA.1 + ?VoteB.1 + ?VoteC.1 + ?VoteD.1 }))
\end{aligned}
$$

BallotB offers to a voter more options than Ballot, and intuitively it should be possible to use a server that guarantees BallotB in place of a server that guarantees Ballot. This is not the case if the contracts are compared with $\sqsubseteq_{\mathrm{SRV}}$, because Ballot $\mathbb{E}_{\mathrm{SRV}}$ BallotB. On the other hand, if we restrict our attention to session contracts, and thus to the pre-order $\varlimsup_{\mathrm{SRV}}^{\mathcal{S C}}$, we have Ballot $\varlimsup_{\mathrm{SRV}}^{\mathcal{S C}}$ BallotB. 
When comparing session contracts relative to this pre-order it will be convenient to work modulo unfolding, which is possible because of the following result:

Proposition 4.5. Let $\sigma_{1}, \sigma_{2}$ be session contracts, then $\sigma_{1} \sqsubseteq_{\mathrm{SRV}}^{\mathcal{S C}} \sigma_{2}$ if and only if $\operatorname{UNFOLD}\left(\sigma_{2}\right) \sqsubseteq \mathcal{S C} \operatorname{SRF} \operatorname{UNFLD}\left(\sigma_{2}\right)$.

Proof. Follows from Proposition 3.22 and 3.23 .

Proposition 4.6. [Bottom element]

The pre-order $\sqsubseteq_{\mathrm{SRV}}^{\mathcal{S C}}$ enjoys the following properties,

(i) it has a has bottom element

(ii) if $\sigma_{\perp}$ is a bottom element of $\sqsubseteq_{\mathrm{SRV}}^{\mathcal{S C}}$ then $\operatorname{UnFOLD}\left(\sigma_{\perp}\right)=\mathbf{1}$

Proof. To prove (i) we show that $\mathbf{1}$ is a bottom element of $\sqsubseteq_{\mathrm{SRV}}^{\mathcal{S C}}$, that is $\mathbf{1} \sqsubseteq_{\mathrm{SRV}}^{\mathcal{S C}} \sigma$ for every session contract $\sigma$. Let $\rho$ be a session contract such that $\rho \dashv \mathbf{1}$. The session contract 1 offers no interaction. Therefore, because of the restricted syntax of session contracts, $\rho$ must also be, modulo unfolding, the simple contract 1. Now fix a session contract $\sigma$. Clearly $\mathbf{1} \dashv \sigma$, therefore from an application of Proposition 3.23 it follows that $\rho \dashv \sigma$.

To prove part (ii) let $\sigma_{\perp}$ be an arbitrary bottom element of $\complement_{\text {SRV }}^{\mathcal{S C}}$. We are required to show that $\operatorname{UNFOLD}\left(\sigma_{\perp}\right)=1$. From the definition of bottom element follows $\sigma_{\perp} \sqsubseteq_{\mathrm{SRV}}^{\mathcal{S C}}$ 1. An application of the previous proposition gives $\operatorname{UNFOLD}\left(\sigma_{\perp}\right) \sqsubseteq_{\mathrm{SRV}}^{\mathcal{S C}} \mathbf{1}$. But now an analysis of the possible syntactic structure of $\operatorname{UNFOLD}\left(\sigma_{\perp}\right)$ quickly yields that it must be $\mathbf{1}$ itself.

Part (ii) is relevant because $\mathbf{1}$ is not the only bottom element; for example it is also true that $\mu X .1 \sqsubseteq_{\mathrm{SRV}}^{\mathcal{S C}} \sigma$ for every $\sigma$.

We now proceed, as in Section 3.2.1. to give a co-inductive characterisation of this more generous pre-order on session contracts, this time taking advantage of their restricted syntactic structure.

Definition 4.7. [Syntactic sub-server relation]

Let $\mathcal{F}_{\preccurlyeq_{\mathrm{SRV}}^{\text {syn }}}: \mathcal{P}\left(\mathcal{S C}^{2}\right) \longrightarrow \mathcal{P}\left(\mathcal{S C}^{2}\right)$ be defined by letting $\left(\sigma_{1}, \sigma_{2}\right) \in \mathcal{F}_{\preccurlyeq_{\mathrm{sRV}}^{\text {syn }}}(\mathcal{R})$ whenever one of the following holds:

(i) $\operatorname{UNFOLD}\left(\sigma_{1}\right)=\mathbf{1}$

(ii) $\operatorname{UNFOLD}\left(\sigma_{2}\right)=? \mathrm{t}_{2} \cdot \sigma_{2}^{\prime}$ and $\operatorname{UnFOLD}\left(\sigma_{1}\right)=? \mathrm{t}_{1} \cdot \sigma_{1}^{\prime}$ with $\mathrm{t}_{1} \preccurlyeq_{\mathrm{g}} \mathrm{t}_{2}$ and $\sigma_{1}^{\prime} \mathcal{R} \sigma_{2}^{\prime}$

(iii) $\operatorname{UnFold}\left(\sigma_{2}\right)=! \mathrm{t}_{2} \cdot \sigma_{2}^{\prime}$ and $\operatorname{UnFOLD}\left(\sigma_{1}\right)=\mathrm{t}_{1} \cdot \sigma_{1}^{\prime}$ with $\mathrm{t}_{2} \preccurlyeq \mathrm{g} \mathrm{t}_{1}$ and $\sigma_{1}^{\prime} \mathcal{R} \sigma_{2}^{\prime}$

(iv) $\operatorname{UNFOLD}\left(\sigma_{2}\right)=\sum_{j \in J} ? 1_{j} \cdot \sigma_{j}^{2}$ and $\operatorname{UNFOLD}\left(\sigma_{1}\right)=\sum_{i \in I} ? 1_{i} \cdot \sigma_{i}^{1}$ with $I \subseteq J$ and $\sigma_{i}^{1} \mathcal{R} \sigma_{i}^{2}$

(v) $\operatorname{Unfold}\left(\sigma_{2}\right)=\bigoplus_{j \in J} ! 1_{j} \cdot \sigma_{j}^{2}$ and $\operatorname{UnFold}\left(\sigma_{1}\right)=\bigoplus_{i \in I} ! 1_{i} \cdot \sigma_{i}^{1}$ with $J \subseteq I$ and $\sigma_{j}^{1} \mathcal{R} \sigma_{j}^{2}$

If $\mathcal{R} \subseteq \mathcal{F}_{\preccurlyeq_{\mathrm{sV}}^{\text {syn }}}(\mathcal{R})$ then we say that $\mathcal{R}$ is a co-inductive syntactic sub-server relation. Let $\preccurlyeq_{\mathrm{SRV}}^{s y n}$ denote the greatest solution of the equation $X=\mathcal{F}_{\preccurlyeq_{\mathrm{SV}} \text { syn }}(X)$. We call this solution the syntactic sub-server. The relation $\preccurlyeq_{\mathrm{SRV}}^{s y n}$ is the greatest co-inductive syntactic sub-server relation. 
We first show that the set based relation $\complement_{\mathrm{SRV}}^{\mathcal{S C}}$ is contained in $\preccurlyeq_{\mathrm{SRV}}^{\text {syn }}$; this will follow if we can show the former satisfies the defining properties of the latter.

Lemma 4.8. Let $\sigma_{1}, \sigma_{2} \in \mathcal{S C}, \sigma_{1}=\operatorname{UnFold}\left(\sigma_{1}\right), \sigma_{2}=\operatorname{UnFOLD}\left(\sigma_{2}\right)$ and $\sigma_{1} \sqsubseteq_{\mathrm{SRV}}^{\mathcal{S C}} \sigma_{2}$. Then

(i) if $\sigma_{1}=! \mathrm{t}_{1} \cdot \sigma_{1}^{\prime}$ then $\sigma_{2}=! \mathrm{t}_{2} \cdot \sigma_{2}^{\prime}, \mathrm{t}_{2} \preccurlyeq \mathrm{g} \mathrm{t}_{1}$ and $\sigma_{1}^{\prime} \sqsubseteq_{\mathrm{SRV}}^{\mathcal{S C}} \sigma_{2}^{\prime}$

(ii) if $\sigma_{1}=? \mathrm{t}_{1} \cdot \sigma_{1}^{\prime}$ then $\sigma_{2}=? \mathrm{t}_{2} \cdot \sigma_{2}^{\prime}, \mathrm{t}_{1} \preccurlyeq \mathrm{g} \mathrm{t}_{2}$ and $\sigma_{1}^{\prime} \sqsubseteq_{\mathrm{SRV}}^{\mathcal{S C}} \sigma_{2}^{\prime}$

(iii) if $\sigma_{1}=\sum_{i \in I} ? 1_{1} \cdot \sigma_{i}^{1}$ then $\sigma_{2}=\sum_{j \in J} ? 1_{j} \cdot \sigma_{j}^{2}, I \subseteq J$ and $\sigma_{i}^{1} \sqsubseteq_{\mathrm{SRV}}^{\mathcal{S C}} \sigma_{i}^{2}$

(iv) if $\sigma_{1}=\bigoplus_{i \in I} ! 1_{i} \cdot \sigma_{i}^{1}$ then $\sigma_{2}=\bigoplus_{j \in J} ! 1_{j} \cdot \sigma_{j}^{2}$ with $J \subseteq I$ and $\sigma_{j}^{1} \sqsubseteq_{\mathrm{SRV}}^{\mathcal{S C}} \sigma_{j}^{2}$

Proof. The proof is by case analysis on the structure of $\sigma_{1}$ and depends greatly on the restricted syntax of session contracts. We give the details of the first case; the others are analogous.

Suppose $\sigma_{1}=! \mathrm{t}_{1} \cdot \sigma_{1}^{\prime}$. Then $? \mathrm{t}_{1} \cdot 1 \dashv \sigma_{1}$ and because $\sigma_{1} \sqsubseteq_{\mathrm{SRV}}^{\mathcal{S C}} \sigma_{2}$ it follows that ? $t_{1} .1 \dashv \sigma_{2}$. Since ? $t_{1} .1$ is stuck, $\sigma_{2}$ has to engage in an action $! t_{2}$ such that ? $t_{1} \bowtie_{c}$ ! $t_{2}$. It follows $t_{2} \preccurlyeq t_{1}$. In reason of the syntax and the the hypothesis $\sigma_{2}=\operatorname{UNFOLD}\left(\sigma_{2}\right)$, the equality $\sigma_{2}=! \mathrm{t}_{2} \cdot \sigma_{2}^{\prime}$ must hold

We also have to prove that $\sigma_{1}^{\prime} \sqsubseteq_{\mathrm{SRV}}^{\mathcal{S C}} \sigma_{2}^{\prime}$. Pick a session contract $\rho$ such that $\rho \dashv \sigma_{1}^{\prime}$. Clearly ? $\mathrm{t}_{1} . \rho \dashv \sigma_{1}$, and thus ? $\mathrm{t}_{1} \cdot \rho \dashv \sigma_{2}$. Since ? $\mathrm{t}_{1} \bowtie_{c} ! \mathrm{t}_{2}$, we apply rule [P-SYNCH] to infer ? $\mathrm{t}_{1} . \rho\left\|\sigma_{2} \stackrel{\tau}{\longrightarrow} \rho\right\| \sigma_{2}^{\prime}$. From the definition of compliance it follows that $\rho \dashv \sigma_{2}^{\prime}$.

Proposition 4.9. For session contracts, $\sigma_{1} \sqsubseteq_{\mathrm{SRV}}^{\mathcal{S C}} \sigma_{2}$ implies $\sigma_{1} \preccurlyeq_{\mathrm{SRV}}^{\text {syn }} \sigma_{2}$.

Proof. We prove that $\sqsubseteq_{\mathrm{SRV}}^{\mathcal{S C}}$ is a pre-fixed point of the function $\mathcal{F}_{\preccurlyeq_{\mathrm{SRV}}^{\text {syn }}}$ of Definition 4.7 that is $\sigma_{1} \sqsubseteq_{\mathrm{SRV}}^{\mathcal{S C}} \sigma_{2}$ implies $\left(\sigma_{1}, \sigma_{2}\right) \in \mathcal{F}_{\preccurlyeq_{\mathrm{SRV}}^{\text {syn }}}\left(\sqsubseteq_{\mathrm{SRV}}^{\mathcal{S C}}\right)$.

Suppose $\sigma_{1} \sqsubseteq_{\mathrm{SRV}}^{\mathcal{S C}} \sigma_{2}$. Then by Proposition 4.5 it follows that $\operatorname{UnFOLD}\left(\sigma_{1}\right) \sqsubseteq_{\mathrm{SRV}}^{\mathcal{S C}}$ $\operatorname{Unfold}\left(\sigma_{2}\right)$. Now if $\operatorname{Unfold}\left(\sigma_{1}\right)=\mathbf{1}$ by definition $\left(\sigma_{1}, \sigma_{2}\right) \in \mathcal{F}_{\preccurlyeq_{\mathrm{SRV}}^{\text {syn }}}\left(\varlimsup_{\mathrm{SRV}}^{\mathcal{S C}}\right)$. Otherwise we can apply Lemma 4.8 to the pair $\operatorname{UNFOLD}\left(\sigma_{1}\right), \operatorname{UNFOLD}\left(\sigma_{2}\right)$. This provides the required information to satisfy the requirements (ii) to (v) in Definition 4.7 , thereby ensuring that $\left(\sigma_{1}, \sigma_{2}\right) \in \mathcal{F}_{\preccurlyeq}^{\text {syn }}\left(\sqsubseteq_{\mathrm{SRV}}^{\mathcal{S C}}\right)$.

Lemma 4.10. Let $\mathcal{R}$ be a co-inductive syntactic sub-server relation and let $\sigma_{1} \mathcal{R} \sigma_{2}$. If $\sigma_{2} \stackrel{\tau}{\longrightarrow} \sigma_{2}^{\prime}$ then $\sigma_{1} \mathcal{R} \sigma_{2}^{\prime}$.

Proof. First note that from Definition 4.7 it follows that

$$
\operatorname{UNFOLD}\left(\sigma_{1}\right) \mathcal{R} \operatorname{UNFOLD}\left(\sigma_{2}\right)
$$

There are two different cases to be discussed, depending on the unfolding of $\sigma_{2}$ being $\sigma_{2}$ itself or not.

(a) If $\operatorname{Unfold}\left(\sigma_{2}\right) \neq \sigma_{2}$ then $\operatorname{UnFold}\left(\sigma_{2}\right)=\operatorname{UnFOLD}\left(\sigma_{2}^{\prime}\right)$. The equality and (6) above imply $\operatorname{UNFOLD}\left(\sigma_{1}\right) \mathcal{R} \operatorname{UNFOLD}\left(\sigma_{2}^{\prime}\right)$; the latter fact means that $\sigma_{1} \mathcal{R} \sigma_{2}^{\prime}$.

(b) If $\operatorname{UNFOLD}\left(\sigma_{2}\right)=\sigma_{2}$ then $\sigma_{2}$ must be an internal sum, say $\sigma_{2}=\bigoplus_{i \in I} ! 1_{\mathrm{i}} \cdot \sigma_{i}^{2}$, because $\sigma_{2}$ can perform a silent move and can not unfold. This implies that $\sigma_{2}^{\prime}$ is the internal sum $\bigoplus_{k \in K} ! 1_{\mathrm{k}} \cdot \sigma_{k}^{2}$ for some $K \subseteq I$. From Definition 4.7 it 
follows that $\operatorname{Unfold}\left(\sigma_{1}\right)=\bigoplus_{j \in J} ! 1_{j} \cdot \sigma_{j}^{2}$ with $I \subseteq J$. Since $\operatorname{UnFoLd}\left(\sigma_{2}^{\prime}\right)=$ $\sigma_{2}^{\prime}$ and $K \subseteq I \subseteq J$ one sees easily that

$$
\operatorname{UNFOLD}\left(\sigma_{1}\right) \mathcal{R} \operatorname{UNFOLD}\left(\sigma_{2}^{\prime}\right)
$$

and thus $\sigma_{1} \mathcal{R} \sigma_{2}$.

Lemma 4.11. Let $\mathcal{R}$ be a co-inductive syntactic sub-server relation. Moreover let $\sigma_{1} \mathcal{R} \sigma_{2}$ and $\sigma_{2} \downarrow \mathrm{R}$. Then $\mathrm{R}^{\prime} \in \operatorname{ACC}\left(\sigma_{1}\right)$ for some $\mathrm{R}^{\prime}$ such that $\mathrm{R}^{\prime} \sqsubseteq \mathrm{R}$.

Proof. Using Lemma 3.2 we know $\operatorname{UnFOLD}\left(\sigma_{2}\right)=\sigma_{2}$, since $\sigma_{2} \stackrel{\tau}{\rightarrow}$. From Definition 4.7 it follows that $\operatorname{UNFOLD}\left(\sigma_{1}\right) \mathcal{R} \sigma_{2}$. Now, according to the cases in Definition 4.7 and a case analysis on the form of $\sigma_{2}$, one can show that $\operatorname{UNFOLD}\left(\sigma_{1}\right) \stackrel{\tau^{\tau}}{\longrightarrow} \sigma_{1}^{\prime}$ for some $\sigma_{1}^{\prime}$ which satisfies the required properties. We leave the details of the case analysis to the reader.

Finally, the proof that $\sigma_{1} \stackrel{\tau}{\longrightarrow}{ }^{*} \sigma_{1}^{\prime}$ amounts in two steps. We apply Lemma 3.2 which ensures that $\sigma_{1} \stackrel{\tau}{\longrightarrow}{ }^{*} \operatorname{UNFOLD}\left(\sigma_{1}\right)$. Now we know that

$$
\sigma_{1} \stackrel{\tau}{\longrightarrow}^{*} \operatorname{UNFOLD}\left(\sigma_{1}\right) \stackrel{\tau}{\longrightarrow} \sigma_{1}^{\prime}
$$

so the transitivity of $\stackrel{\tau}{\longrightarrow}{ }^{*}$ gives the result.

Lemma 4.10 and Lemma 4.11 proves that the pre-order $\preccurlyeq_{\mathrm{SRV}}^{\text {syn }}$ enjoys two of the properties of the pre-order $\preccurlyeq_{\mathrm{SRV}}$. The third property of $\preccurlyeq_{\mathrm{SRV}}$, though, does not hold.

Example 4.12. Let $\sigma_{1}=? 1_{1} .1$ and $\sigma_{2}=? l_{1} \cdot 1+? 1_{2} .1$ Recall that $\sigma_{1} \preccurlyeq_{\text {SRV }}^{\text {syn }} \sigma_{2}$. Then $\sigma_{2} \stackrel{? 1_{2}}{\longrightarrow} 1$ and $! 1_{2} \bowtie_{c} ? 1_{2}$, but $\left(\sigma_{1}\right.$ AFTER $\left.! 1_{2}\right)=\emptyset$. As in Example 4.3 the crucial fact is that the pre-order $\preccurlyeq_{\mathrm{SRV}}^{s y n}$ allows implementation refinement Pad10.

Theorem 4.13. [Co-inductive characterisation] For session contracts $\sigma_{1}, \sigma_{2}$, $\sigma_{1} \sqsubseteq_{\mathrm{SRV}} \sigma_{2}$ if and only if $\sigma_{1} \preccurlyeq_{\mathrm{SRV}}^{\text {syn }} \sigma_{2}$.

Proof. The only if part of the theorem is Proposition 4.9 while the if part, that is the set inclusion $\preccurlyeq_{\mathrm{SRV}}^{s y n} \subseteq \sqsubseteq_{\mathrm{SRV}}^{\mathcal{S C}}$, follows from the fact that the relation

$$
\mathcal{R} \triangleq\left\{\left(\rho, \sigma_{2}\right) \mid \sigma_{1} \preccurlyeq_{\mathrm{SRV}}^{\text {syn }} \sigma_{2}, \rho \dashv \sigma_{1} \text { for some } \sigma_{1} \in \mathcal{S C}\right\}
$$

contains $\left(\rho, \sigma_{2}\right)$ and is a compliance. We prove the latter.

We have to show that $\mathcal{R}$ satisfies the two properties in Definition 3.16 . Let $(\rho, \sigma) \in \mathcal{R}$; by definition there exists a $\sigma_{1}$ such that $\rho \dashv \sigma_{1}$ and $\sigma_{1} \preccurlyeq_{\mathrm{SRV}}^{s y n} \sigma$.

To prove point (i) of Definition 3.16 assume $\rho \| \sigma \stackrel{\tau}{\nrightarrow}$. This implies that $\sigma$ and $\rho$ are both stuck, so $\operatorname{ACC}(\rho)=\{\mathrm{s}\}$ and $\operatorname{ACC}(\sigma)=\{\mathrm{R}\}$, and that

$$
\alpha \in \mathrm{R} \text { implies } \beta \not \oiint_{c} \alpha \text { whenever } \beta \in \mathrm{S}
$$

An application of Lemma 4.11 and of the definition of acceptance set gives a $\sigma_{1}^{\prime}$ such that $\sigma_{1} \stackrel{\tau}{\longrightarrow} \sigma_{1}^{\prime} \downarrow \mathrm{R}^{\prime}$ and $\mathrm{R}^{\prime} \sqsubseteq \mathrm{R}$. The last inequality implies that

$$
\alpha \in \mathrm{R}^{\prime} \text { implies } \beta ぬ_{c} \alpha \text { whenever } \beta \in \mathrm{S}
$$


therefore, $\rho \| \sigma_{1}^{\prime} \stackrel{\tau}{\hookrightarrow}$. Part (ii) of Definition 3.16 and the assumption $\rho \dashv \sigma_{1}$ imply that $\rho$ complies with $\sigma_{1}^{\prime}$ so $\rho \stackrel{\checkmark}{\longrightarrow}$.

What we have left to do now is to show that if $\rho\left\|\sigma \stackrel{\tau}{\longrightarrow} \rho^{\prime}\right\| \sigma^{\prime}$ then $\left(\rho^{\prime}, \sigma^{\prime}\right) \in \mathcal{R}$, that is there exists a $\hat{\sigma} \in \mathcal{S C}$ such that

$$
\rho^{\prime} \dashv \hat{\sigma}, \quad \hat{\sigma} \preccurlyeq_{\mathrm{SRV}}^{\text {syn }} \sigma^{\prime}
$$

Assume $\rho\left\|\sigma \stackrel{\tau}{\longrightarrow} \rho^{\prime}\right\| \sigma^{\prime}$. The argument depends on the rule used to infer this silent move (see Figure 7). If rule [P-SIL-L] was used then $\sigma^{\prime}=\sigma$ and $\rho \stackrel{\tau}{\longrightarrow} \rho^{\prime}$; let $\hat{\sigma}=\sigma_{1}$. Then we already know that $\hat{\sigma} \preccurlyeq_{\mathrm{SRv}}^{s y n} \sigma^{\prime}$, and part (ii) of Definition 3.16 implies $\rho^{\prime} \dashv \hat{\sigma}$. If rule [P-SIL-R] was applied then $\rho^{\prime}=\rho$ and $\sigma \stackrel{\tau}{\longrightarrow} \sigma^{\prime}$. In this case an application of Lemma 4.10 implies $\sigma_{1} \preccurlyeq_{\mathrm{SRV}}^{\text {syn }} \sigma^{\prime}$. We know by assumption that $\rho \dashv \sigma_{1}$, so the $\hat{\sigma}$ we are looking for is $\sigma_{1}$.

If rule $[\mathrm{P}-\mathrm{SYNCH}]$ was applied then

$$
\rho \stackrel{\alpha}{\longrightarrow} \rho^{\prime}, \quad \sigma \stackrel{\beta}{\longrightarrow} \sigma^{\prime}, \quad \alpha \bowtie_{c} \beta .
$$

Since $\rho$ performs an observable action part (iii) of Lemma 4.1 implies $\rho \stackrel{\checkmark}{\sim}$.

Let us turn our attention to $\operatorname{UNFOLD}\left(\sigma_{1}\right)$. The assumption $\rho \dashv \sigma_{1}$ together with Proposition 3.22 implies that (a) $\rho \dashv \operatorname{UNFOLD}\left(\sigma_{1}\right)$. The assumption $\sigma_{1} \preccurlyeq_{\mathrm{SRV}}^{\text {syn }}$ $\sigma$ and Definition 4.7 imply that (b) $\operatorname{UNFOLD}\left(\sigma_{1}\right) \preccurlyeq_{\text {SRV }}^{\text {syn }} \sigma$.

We know that $\rho \dashv \operatorname{UNFOLD}\left(\sigma_{1}\right)$ ((a) above), and that $\rho \stackrel{\checkmark}{4}$; together with part (ii), these facts force $\operatorname{UNFOLD}\left(\sigma_{1}\right)$ to offer an action $\delta$ such that $\delta \bowtie_{c} \alpha$. Thus, for some $\sigma_{1}^{\prime}$,

$$
\operatorname{UNFOLD}\left(\sigma_{1}\right) \stackrel{\delta}{\longrightarrow} \sigma_{1}^{\prime}
$$

An application of rule [P-SYNCH] ensures that

$$
\rho\left\|\operatorname{UNFOLD}\left(\sigma_{1}\right) \stackrel{\tau}{\longrightarrow} \rho^{\prime}\right\| \sigma_{1}^{\prime}
$$

Now (a) and part (ii) of Definition 3.16 imply that $\rho^{\prime} \dashv \sigma_{1}^{\prime}$. We choose $\sigma_{1}^{\prime}$ as candidate $\hat{\sigma}$.

To finish the proof we have to show that $\sigma_{1}^{\prime} \preccurlyeq_{\mathrm{SRV}}^{\text {syn }} \sigma^{\prime}$. The argument is a case analysis on the action $\beta$. Four cases are to be discussed, but, as they are all similar, we give a detailed account only of two of them.

If $\beta=$ ? $\mathrm{t}_{2}$ then (b) above and case (ii) of Definition 4.7 ensure that $\sigma^{\prime}$ is unique, and so is $\sigma_{1}^{\prime}$ as well. The same definition implies also that $\sigma_{1}^{\prime} \preccurlyeq_{\text {SRV }}^{\text {syn }} \sigma^{\prime}$.

If, for some label $1, \beta=$ ? 1 then the definition of $\bowtie_{c}$ implies that $\alpha=! 1$, and the assumption $\delta \bowtie_{c} \alpha$ implies $\delta=$ ?1. We have proven that $\delta=\beta$. Now (b) above and case (iv) of Definition 4.7 imply that $\sigma_{1}^{\prime} \preccurlyeq_{\mathrm{SRV}}^{\text {syn }} \sigma^{\prime}$.

We conclude this subsection with a summary of our knowledge on the preorders which compare contracts on the server side of the compliance relation.

Corollary 4.14. The following equalities and inequality hold

$$
\sqsubseteq_{\mathrm{MUST}}=\sqsubseteq_{\mathrm{SRV}} \neq \sqsubseteq_{\mathrm{SRV}}^{\mathcal{S C}}=\preccurlyeq_{\mathrm{SRV}}^{\text {syn }}
$$

Proof. It is a consequence of Corollary 3.49 . Example 4.3 and Theorem 4.13 . 


\subsection{The client pre-order}

We introduce a new pre-order which compares the capacity of clients to be satisfied by servers. The structure of this sub-section is similar to that of the previous one on the restricted server pre-order.

Definition 4.15. [Restricted client pre-order]

For $\rho_{1}, \rho_{2} \in \mathcal{S C}$ let $\rho_{1} \sqsubseteq_{\mathrm{CLT}}^{\mathcal{S C}} \rho_{2}$ whenever $\rho_{1} \dashv \sigma$ implies $\rho_{2} \dashv \sigma$ for every $\sigma$ in $\mathcal{S C}$.

Also the restricted client pre-order let us reason modulo unfolding.

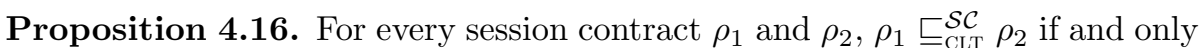
if $\operatorname{UNFOLD}\left(\rho_{2}\right) \sqsubseteq_{\mathrm{CLT}}^{\mathcal{S C}} \operatorname{UNFOLD}\left(\rho_{2}\right)$.

Proof. Follows from Proposition 3.22 and 3.23 .

Example 4.17. We have argued in Example 4.3 that $? \mathfrak{l}_{1} \cdot \mathbf{1} \sqsubseteq_{\mathrm{SRV}}^{\mathcal{S C}} ? \mathfrak{l}_{2} \cdot \mathbf{1}+? \mathfrak{l}_{1} \cdot \mathbf{1}$. A similar argument, this time applied to server-side session contracts, can be used to show that

$$
? 1_{1} \cdot 1 \sqsubseteq_{\text {CLT }}^{\mathcal{S C}} ? 1_{2} \cdot ? 1_{2} \cdot 1+? 1_{1} \cdot 1
$$

Similarly to what happen for server contracts, if we turn our attention to general contracts then the session contracts above are no longer related. Let us see why. The client $? 1_{1} . \mathbf{1}$ complies with the server $! 1_{1} . \mathbf{1}+! 1_{2} . \mathbf{1}$, because the action $! 1_{2}$ will never be performed by the server. On the other hand

$$
? 1_{2} . ? 1_{2} .1+? 1_{1} .1\left\|! 1_{1} .1+! 1_{2} .1 \stackrel{\tau}{\longrightarrow} ? 1_{2} .1\right\| 1 \stackrel{\tau}{\not}
$$

and $? \mathfrak{I}_{2} .1 \stackrel{\checkmark}{\sim}$; this proves that

$$
? 1_{2} \cdot ? 1_{2} \cdot 1+? 1_{1} .1 \nmid ! 1_{1} \cdot 1+! 1_{2} \cdot 1
$$

Had we defined in the obvious way the pre-order $\sqsubseteq_{\text {CLT }}$ on contracts, then the argument above would have proven that

$$
! 1_{1} \cdot 1 \nsubseteq \mathrm{CLT} ? 1_{2} .1 \stackrel{\checkmark}{\sim}
$$

We have therefore shown that

$$
\sqsubseteq_{\mathrm{CLT}}^{\mathcal{S C}} \nsubseteq \coprod_{\mathrm{CLT}}
$$

We have seen in Proposition 4.6 that the session contract $\mathbf{1}$ is a bottom element in the restricted server pre-order. The client pre-order enjoys the dual property.

Proposition 4.18. [Top element]

The pre-order $\complement_{\mathrm{CLT}}^{\mathcal{S C}}$ enjoys the following two properties,

(i) it has a top element

(ii) if $\sigma_{\top}$ is a top element of $\sqsubseteq_{\text {CLT }}^{\mathcal{S C}}$ then $\operatorname{UnfOLD}\left(\sigma_{\top}\right)=\mathbf{1}$ 
Proof. Since $\mathbf{1} \dashv \sigma$ for every contract $\sigma$, the session contract $\mathbf{1}$ it is a top element in the restricted client pre-order. Moreover, reasoning as in Proposition 4.6 we can show that if $\sigma_{\top}$ is an arbitrary top element then $\operatorname{UnFOLD}\left(\sigma_{\top}\right)=\mathbf{1}$.

Definition 4.19. [Syntactic sub-client relation]

Let $\mathcal{F}_{\preccurlyeq_{\text {cLT }}^{\text {syn }}}: \mathcal{P}\left(\mathcal{S C}^{2}\right) \longrightarrow \mathcal{P}\left(\mathcal{S C}^{2}\right)$ be defined so that $\left(\rho_{1}, \rho_{2}\right) \in \mathcal{F}_{\preccurlyeq_{\text {CLT }}^{\text {syn }}}(\mathcal{R})$ whenever one of the following is true:

(i) $\operatorname{UNFOLD}\left(\rho_{2}\right)=\mathbf{1}$

(ii) $\operatorname{UNFOLD}\left(\rho_{2}\right)=? \mathrm{t}_{2} \cdot \rho_{2}^{\prime}$ and $\operatorname{UNFOLD}\left(\rho_{1}\right)=$ ? $\mathrm{t}_{1} \cdot \rho_{1}^{\prime}$ with $\mathrm{t}_{1} \preccurlyeq_{\mathrm{g}} \mathrm{t}_{2}$ and $\rho_{1}^{\prime} \mathcal{R} \rho_{2}^{\prime}$

(iii) $\operatorname{UNFOLD}\left(\rho_{2}\right)=! \mathrm{t}_{2} \cdot \rho_{2}^{\prime}$ and $\operatorname{UNFOLD}\left(\rho_{1}\right)=! \mathrm{t}_{1} \cdot \rho_{1}^{\prime}$ with $\mathrm{t}_{2} \preccurlyeq \mathrm{g} \mathrm{t}_{1}$ and $\rho_{1}^{\prime} \mathcal{R} \rho_{2}^{\prime}$

(iv) $\operatorname{UNFOLD}\left(\rho_{2}\right)=\sum_{j \in J} 1_{j} \cdot \rho_{j}^{2}$ and $\operatorname{UNFOLD}\left(\rho_{1}\right)=\sum_{i \in I} 1_{i} \cdot \rho_{i}^{1}$ with $I \subseteq J$ and $\rho_{i}^{1} \mathcal{R} \rho_{i}^{2}$

(v) $\operatorname{UNFOLD}\left(\rho_{2}\right)=\bigoplus_{j \in J} \mathrm{l}_{j} \cdot \sigma_{j}^{2}$ and $\operatorname{UNFOLD}\left(\rho_{1}\right)=\bigoplus_{i \in I} \mathrm{l}_{i} \cdot \sigma_{i}^{1}$ with $J \subseteq I$ and $\sigma_{j}^{1} \mathcal{R} \sigma_{j}^{2}$

If $\mathcal{R} \subseteq \mathcal{F} \preccurlyeq_{\text {cyn }}^{\text {syn }}(\mathcal{R})$ then we say that $\mathcal{R}$ is a co-inductive syntactic sub-clientrelation. Let $\preccurlyeq_{\text {CLT }}^{\text {syn }}$ denote the greatest solution of the equation $X=\mathcal{F}_{\preccurlyeq_{\text {cLT }}^{\text {syn }}}(X)$. We call this solution the sub-clientrelation. The relation $\preccurlyeq_{\text {CLT }}^{s y n}$ is the greatest coinductive syntactic sub-clientrelation.

Lemma 4.20. Let $\rho_{1}, \rho_{2} \in \mathcal{S C}, \rho_{1}=\operatorname{UNFOLD}\left(\rho_{1}\right), \rho_{2}=\operatorname{UNFOLD}\left(\rho_{2}\right)$ and

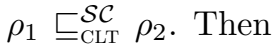

(i) if $\rho_{2}=! \mathrm{t}_{2} \cdot \rho_{2}^{\prime}$ then $\rho_{1}=! \mathrm{t}_{1} \cdot \rho_{1}^{\prime}, \mathrm{t}_{2} \preccurlyeq \mathrm{g} \mathrm{t}_{1}$ and $\rho_{1}^{\prime} \sqsubseteq_{\text {CLT }}^{\mathcal{S C}} \rho_{2}^{\prime}$

(ii) if $\rho_{2}=? \mathrm{t}_{2} \cdot \rho_{2}^{\prime}$ then $\rho_{1}=? \mathrm{t}_{1} \cdot \rho_{1}^{\prime}, \mathrm{t}_{1} \preccurlyeq \mathrm{g} \mathrm{t}_{2}$ and $\rho_{1}^{\prime} \sqsubseteq_{\text {CLT }}^{\mathcal{S C}} \rho_{2}^{\prime}$

(iii) if $\rho_{2}=\sum_{j \in J} ? 1_{j} \cdot \rho_{j}^{2}$ then $\rho_{1}=\sum_{i \in I} ? 1_{i} \cdot \rho_{i}^{1}$ with $I \subseteq J$ and $\rho_{i}^{1} \sqsubseteq_{\text {CLT }}^{\mathcal{S C}} \rho_{i}^{2}$

(iv) if $\rho_{2}=\bigoplus_{j \in J} ! 1_{j} \cdot \rho_{j}^{2}$ then $\rho_{1}=\bigoplus_{i \in I} ! 1_{i} \cdot \rho_{i}^{1}$ with $J \subseteq I$ and $\rho_{j}^{1} \sqsubseteq_{\text {CLT }}^{\mathcal{S C}} \rho_{j}^{2}$

Proof. The proof is almost the same of lemma 4.8, the difference being that here we look at left-hand side of the compliance relation.

Proposition 4.21. For every session contract $\rho_{1}$ and $\rho_{2}$, if $\rho_{1} \sqsubseteq_{\mathrm{CLT}}^{\mathcal{S C}} \rho_{2}$ then $\rho_{1} \preccurlyeq_{\text {CLT }}^{\text {syn }} \rho_{2}$.

Proof. The argument is similar to the one of Proposition 4.9 , but here we use the function $\mathcal{F}_{\preccurlyeq \text { syn }}^{\text {syn }}$ and Lemma 4.20

Lemma 4.22. Let $\mathcal{R}$ be a co-inductive sub-client relation and let $\rho_{1} \preccurlyeq_{\text {CLT }}^{\text {syn }} \rho_{2}$. If $\rho_{2} \stackrel{\tau}{\longrightarrow} \rho_{2}^{\prime}$ then $\rho_{1} \preccurlyeq \preccurlyeq_{\text {CLT }}^{\text {syn }} \rho_{2}^{\prime}$.

Proof. The proof is similar to the proof of Lemma 4.10

Theorem 4.23. [Co-inductive characterisation]

Let $\rho, \sigma \in \mathcal{S C}$. Then $\rho \preccurlyeq_{\text {CLT }}^{s y n} \sigma$ if and only if $\rho \sqsubseteq_{\text {CLT }}^{\mathcal{S C}} \sigma$. 
Proof. In view of Proposition 4.21 we have to prove only the inclusion $\preccurlyeq_{\text {CLT }}^{\text {syn } \subseteq}$ $\sqsubseteq_{\text {CLT }}^{\mathcal{S C}}$. It is enough to show that

$$
\mathcal{R} \triangleq\left\{\left(\rho_{2}, \sigma\right) \mid \rho_{1} \preccurlyeq_{\mathrm{CLT}}^{\text {syn }} \rho_{2}, \rho_{1} \dashv \sigma \text { for some } \rho_{1} \in \mathcal{S C}\right\}
$$

is a co-inductive compliance. Let $(\rho, \sigma) \in \mathcal{R}$; by definition of $\mathcal{R}$ there exists a $\rho_{1}$ such that $\rho_{1} \preccurlyeq_{\text {CLT }}^{\text {syn }} \rho$ and $\rho_{1} \dashv \sigma$.

We prove part (i) of Definition 3.16. Assume $\rho \| \sigma \stackrel{\tau}{\digamma}$; we have to show that $\rho \stackrel{\checkmark}{\longrightarrow}$.

To this aim it is sufficient to show

$$
\operatorname{UNFOLD}\left(\rho_{1}\right)=\mathbf{1}
$$

We explain why this fact suffice. Assume (6). Since $\operatorname{UnFOLD}\left(\rho_{1}\right) \preccurlyeq_{\text {CLT }}^{\text {syn }} \rho$ we know that $\left(\rho_{1}, \rho\right) \in \mathcal{F}_{\preccurlyeq_{\text {CLT }}^{\text {syn }}}\left(\preccurlyeq_{\text {CLT }}^{\text {syn }}\right)$. This is possible only thanks to case (i) of Definition 4.19, and therefore $\operatorname{Unfold}(\rho)=1$. Since $\rho \stackrel{\tau}{\dagger}$, part (i) of Lemma 3.2 implies $\rho=\operatorname{UNFOLD}(\rho)$, and so, now, an application of part (ii) of Lemma 4.1 ensures $\rho \stackrel{\checkmark}{\longrightarrow}$.

We prove 60. The argument revolves around the unfolding of $\rho_{1}$. To begin with, note two facts: one, the assumption $\rho_{1} \preccurlyeq_{\text {CLT }}^{\text {syn }} \rho$ and Definition 4.19 imply $\operatorname{UNFOLD}\left(\rho_{1}\right) \preccurlyeq_{\mathrm{CLT}}^{s y n} \rho$; and the other, the assumption $\rho_{1} \dashv \sigma$ and Proposition 3.22 imply $\operatorname{UNFOLD}\left(\rho_{1}\right) \dashv \sigma$.

The fact that $\rho \| \sigma \stackrel{\tau}{\leftrightarrow}$ can be used to prove

$$
\alpha \in \mathrm{R} \text { implies } \alpha \not \not_{c} \beta \text { for every } \beta \in \mathrm{S}
$$

From the definition of acceptance set and $\rho_{1} \stackrel{\tau}{\longrightarrow} * \operatorname{UNFOLD}\left(\rho_{1}\right)$ (part (ii) of Lemma 3.2 it follows

$$
\operatorname{ACC}\left(\operatorname{UnFOLD}\left(\rho_{1}\right)\right) \subseteq \operatorname{ACC}\left(\rho_{1}\right)
$$

Now we prove that $\operatorname{UnFOLD}\left(\rho_{1}\right)=\mathbf{1}$. Fix a stuck derivative $\rho_{1}^{\prime}$ of $\operatorname{UNFOLD}\left(\rho_{1}\right)$ :

$$
\operatorname{UNFOLD}\left(\rho_{1}\right) \stackrel{\tau}{\longrightarrow} \rho_{1}^{\prime} \stackrel{\tau}{\longrightarrow}
$$

Such a stuck state exists because of the restricted syntax of session contracts. Further, since $\rho_{1} \stackrel{\tau}{\nrightarrow}$, by definition we have $\rho_{1} \downarrow$ R for some R. Point 8 implies

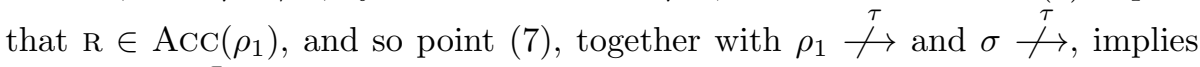
that $\rho_{1}^{\prime} \| \sigma \stackrel{\tau}{\hookrightarrow}$. The fact that $\operatorname{UnFOLD}\left(\rho_{1}\right) \dashv \sigma$ and part (ii) of Definition 3.16 now imply that $\rho_{1}^{\prime} \stackrel{\checkmark}{\longrightarrow}$. We can now apply part (ii) of Lemma 4.1 to obtain $\operatorname{UNFOLD}\left(\rho_{1}\right)=\mathbf{1}$.

As yet we have proven that $(\rho, \sigma)$ respects part (i) of Definition 3.16. The argument to show that also part (ii) of Definition 3.16 holds is similar to the one used in Theorem 4.13. The difference amounts to the use of Definition 4.19 in place of Definition 4.7. We leave the details to the reader. 


\section{$5 \quad$ Modelling session types}

The interpretation of session types as contracts is expressed as a function from the language $L_{\mathcal{S} \mathcal{T}}$ in Section 2 to the language $L_{\mathcal{S C}}$ in Section 4 . The function is little more than a syntactic transformation.

Let $\mathcal{M}: L_{\mathcal{S T}} \longrightarrow L_{\mathcal{S C}}$ be defined by:

$$
\mathcal{M}(S)= \begin{cases}\mathbf{1} & \text { if } S=\mathrm{END} \\ \operatorname{lt} . \mathcal{M}(S) & \text { if } S=![\mathrm{t}] ; S \\ ? \mathrm{t} . \mathcal{M}(S) & \text { if } S=?[\mathrm{t}] ; S \\ \sum_{i \in[1 ; n]} ? 1_{i} \cdot \mathcal{M}\left(S_{i}\right) & \text { if } S=\&\left\langle 1_{1}: S_{1}, \ldots, 1_{n}: S_{n}\right\rangle \\ \bigoplus_{i \in[1 ; n]} ! 1_{i} \cdot \mathcal{M}\left(S_{i}\right) & \text { if } S=\oplus\left\langle 1_{1}: S_{1}, \ldots, 1_{n}: S_{n}\right\rangle \\ \mu x \cdot \mathcal{M}\left(S^{\prime}\right) & \text { if } S=\mu X . S^{\prime} \\ x & \text { if } S=X\end{cases}
$$

It is easy to see that $\mathcal{M}$ maps session types, $\mathcal{S T}$, to session contracts, $\mathcal{S C}$; indeed it defines a bijection between these sets:

- for every $\sigma \in \mathcal{S C}$ there exists some session type $T$ such that $\mathcal{M}(T)=\sigma$

- if $\mathcal{M}\left(T_{1}\right)=\mathcal{M}\left(T_{2}\right)$ then $T_{1}=T_{2}$

where $T_{1}=T_{2}$ denotes syntactic identity. Further, substitution is preserved by $\mathcal{M}$.

Lemma 5.1. Let $S, T \in \mathcal{S} \mathcal{T}$. Then $\mathcal{M}\left(S\left\{{ }^{T} / X\right\}\right)=(\mathcal{M}(S))\{\mathcal{M}(T) / \mathcal{M}(X)\}$.

Proof. The proof is by structural induction on S.

The interpretation also commutes with the two functions $\operatorname{dpt}(-)$ and $\operatorname{UNFOLD}(-)$ :

Lemma 5.2. For every $T \in \mathcal{S T}$ and $\sigma \in \mathcal{S C}$

(i) $\operatorname{dpt}(T)=\operatorname{dpt}(\mathcal{M}(T))$

(ii) $\operatorname{UNFOLD}(\mathcal{M}(T))=\mathcal{M}(\operatorname{UNFOLD}(T))$

(iii) $\operatorname{UNFold}\left(\mathcal{M}^{-1}(\sigma)\right)=T$ if and only if $\operatorname{UNFOLD}(\sigma)=\mathcal{M}(T)$

Proof. The proofs of the first two points are by induction on $\operatorname{dpt}(T)$, the proof of (ii) using (i) and the previous lemma. The third point follows immediately from (ii).

As we have shown, the difficulty is to find a natural pre-order on session contracts which accurately reflects the sub-typing relation on session contracts. There are two obvious candidates, the restricted server pre-order and the restricted client pre-order on session contracts. The difficulty lies in the interpretation of END.

Example 5.3. Recall that $\mathcal{M}(\mathrm{END})=1$. In the restricted server pre-order the session contract $\mathbf{1}$ is a least element, being smaller or equal to every other session contract. On the other hand, for session types END $\preccurlyeq_{\mathrm{ST}} T$ if and only 
if $\operatorname{UNFOLD}(T)=$ END. Consequently the relation $\complement_{\mathrm{SRV}}^{\mathcal{S C}}$ is an unsound model for sub-typing between session types. For example:

$$
\mathbf{1} \sqsubseteq_{\mathrm{SRV}}^{\mathcal{S C}} ! \mathrm{t} . \mathbf{1}, \quad \mathrm{END} \nprec_{\mathrm{ST}} ![\mathrm{t}] ; \mathrm{END}
$$

The restricted client pre-order presents the dual issue as it relates every session contract to $\mathbf{1}$; it is one of the top element. Once again a model based on $\sqsubseteq$ CLT would be unsound:

$$
\text { !t.1 } \varlimsup_{\mathrm{CLT}}^{\mathcal{S C}} \mathbf{1}, \quad ![\mathrm{t}] ; \mathrm{END} \nprec_{\mathrm{ST}} \quad \mathrm{END}
$$

The main result of the paper is that the bijection $\mathcal{M}$ gives a fully abstract interpretation of sub-typing between session types in terms of session contracts, provided we combine these two set-based pre-orders.

Definition 5.4. [Session contract pre-order]

For $\sigma_{1}, \sigma_{2} \in \mathcal{S C}$ let $\sigma_{1} \sqsubseteq^{\mathcal{S C}} \sigma_{2}$ whenever $\sigma_{1} \sqsubseteq_{\mathrm{SRV}}^{\mathcal{S C}} \sigma_{2}$ and $\sigma_{1} \sqsubseteq_{\mathrm{CLT}}^{\mathcal{S C}} \sigma_{2}$.

Example 5.5. It is instructive to see the behaviour of $\mathbf{1}$, the image of END under $\mathcal{M}$, relative to this combined pre-order. First suppose $\sigma \sqsubseteq^{\mathcal{S C}} \mathbf{1}$ for some session contract $\sigma$. This implies $\sigma \sqsubseteq_{\mathrm{SRV}}^{\mathcal{S C}} \mathbf{1}$ and therefore, as we have shown in Proposition 4.6, $\sigma$ must be a bottom element relative to $\complement_{\mathrm{SRV}}^{\mathcal{S C}}$ and $\operatorname{UNFOLD}(\sigma)$ must be 1. A similar argument, using the pre-order $\square_{\text {CLT }}^{\mathcal{S C}}$ ensures that if $\mathbf{1} \sqsubseteq^{\mathcal{S C}} \sigma$ then $\operatorname{UNFOLD}(\sigma)$ must also be $\mathbf{1}$.

In other words modulo unfolding the only session contract related to $\mathbf{1}$ via $\complement^{\mathcal{S C}}$ is $\mathbf{1}$ itself.

Proposition 5.6. [Completeness]

For session contracts, $\sigma_{1} \sqsubseteq^{\mathcal{S C}} \sigma_{2}$ implies $\mathcal{M}^{-1}\left(\sigma_{1}\right) \preccurlyeq_{\mathrm{ST}} \mathcal{M}^{-1}\left(\sigma_{2}\right)$.

Proof. Let $\mathcal{R}$ be the relation over session types defined by

$$
\mathcal{R} \triangleq\left\{\left(\mathcal{M}^{-1}\left(\sigma_{1}\right), \mathcal{M}^{-1}\left(\sigma_{2}\right)\right) \mid \sigma_{1} \preccurlyeq_{\mathrm{SRV}}^{\text {syn }} \sigma_{2}, \sigma_{1} \preccurlyeq_{\mathrm{CLT}}^{\text {syn }} \sigma_{2}\right\}
$$

By showing the $\mathcal{R}$ is a type simulation, that is it satisfies the properties given in Definition 2.7, the result will follow because of Theorems 4.13, and 4.23.

The proof proceeds by a case analysis on the structure of $\operatorname{UNFOLD}\left(\sigma_{1}\right)$; we give the details of two of them.

- Suppose Unfold $\left(\mathcal{M}^{-1}\left(\sigma_{1}\right)\right)=$ End. According to Definition 2.7 we have to show that

$$
\operatorname{UNFOLD}\left(\mathcal{M}^{-1}(\sigma)\right)=\operatorname{END} .
$$

Because of part (iii) of Lemma 5.2 we know that $\operatorname{UNFOLD}\left(\sigma_{1}\right)=\mathbf{1}$; moreover in Example 5.5 above we have already reasoned that $\operatorname{UNFOLD}\left(\sigma_{2}\right)$ must be 1 .

- Suppose $\operatorname{Unfold}\left(\mathcal{M}^{-1}\left(\sigma_{1}\right)\right)=!\left[\mathrm{t}_{1}\right] ; S_{1}$. We are required to prove that

$$
\operatorname{UNFOLD}\left(\mathcal{M}^{-1}\left(\sigma_{2}\right)\right)=!\left[\mathrm{t}_{2}\right] ; S_{2},
$$

for some $\mathrm{t}_{2}$ and $S_{2}$ such that $\mathrm{t}_{2} \preccurlyeq_{\mathrm{g}} \mathrm{t}_{1}$ and $\left(\mathcal{M}\left(S_{1}\right), \mathcal{M}\left(S_{2}\right)\right) \in \preccurlyeq_{\mathrm{SRV}}^{\text {syn }} \cap \preccurlyeq_{\text {CLT }}^{\text {syn }}$. 
Again by Lemma 5.2 (iii) we know that $\operatorname{UNFOLD}\left(\sigma_{1}\right)=! \mathrm{t}_{1} \cdot \mathcal{M}\left(S_{1}\right)$. Using the fact that $\sigma_{1} \preccurlyeq_{\mathrm{SRV}}^{s y n} \sigma_{2}$, and by Definition 4.7, we know that $\operatorname{UNFOLD}\left(\sigma_{2}\right)=$ $! \mathrm{t}_{2} . \sigma_{2}^{\prime}$ for some $\mathrm{t}_{1}$ such that $\mathrm{t}_{2} \preccurlyeq_{\mathrm{g}} \mathrm{t}_{1}$ and $\mathcal{M}\left(S_{1}\right) \preccurlyeq_{\mathrm{SRV}}^{\text {syn }} \sigma_{2}^{\prime}$. Now letting $S_{2}$ denote $\mathcal{M}^{-1}\left(\sigma_{2}^{\prime}\right)$, another application of Lemma 5.2 (iii) ensures that $(90$ above is satisfied. By the definition of $S_{2}$ we also have the requirement $\mathcal{M}\left(S_{1}\right) \preccurlyeq_{\mathrm{SRV}}^{\text {syn }} \mathcal{M}\left(S_{2}\right)$.

It remains to show $\mathcal{M}\left(S_{1}\right) \preccurlyeq_{\mathrm{CLT}}^{\text {syn }} \mathcal{M}\left(S_{2}\right)$. But this follows from $\sigma_{1} \preccurlyeq_{\mathrm{CLT}}^{\text {syn }} \sigma_{2}$, by part (iii) of Definition 4.19

The proof for the remaining cases are similar and left to the reader.

Theorem 5.7. [Full abstraction]

For all session types, $T_{1} \preccurlyeq_{\mathrm{ST}} T_{2}$ if and only if $\mathcal{M}\left(T_{1}\right) \sqsubseteq^{\mathcal{S C}} \mathcal{M}\left(T_{2}\right)$.

Proof. Thanks to the completeness theorem, Theorem 5.6, it is sufficient to prove that $T_{1} \preccurlyeq_{\mathrm{ST}} T_{2}$ implies $\mathcal{M}\left(T_{1}\right) \sqsubseteq_{\mathrm{SRV}}^{\mathcal{S C}} \mathcal{M}\left(T_{2}\right)$ and $\mathcal{M}\left(T_{1}\right) \sqsubseteq_{\mathrm{CLT}}^{\mathcal{S C}} \mathcal{M}\left(T_{2}\right)$. As an example we outline the proof of the former. Because of Theorem 4.13 it is sufficient to show that the relation $\mathcal{R}$ given by

$$
\mathcal{R}=\left\{\left(\sigma_{1}, \sigma_{2}\right) \mid \mathcal{M}^{-1}\left(\sigma_{1}\right) \preccurlyeq{ }_{\mathrm{ST}} \mathcal{M}^{-1}\left(\sigma_{2}\right)\right\}
$$

is a syntactic sub-server relation, that is $\mathcal{R} \subseteq \mathcal{F}_{\preccurlyeq_{\mathrm{sV}}^{\text {syn }}}(\mathcal{R})$, where $\mathcal{F}_{\preccurlyeq_{\mathrm{sV}}^{\text {syn }}}$ is given in Definition 4.7

Suppose $\left(\sigma_{1}, \sigma_{2}\right) \in \mathcal{R}$. The proof is a case analysis.

- If $\operatorname{UnfOLD}\left(\sigma_{1}\right)=\mathbf{1}$ we have nothing to prove because condition (i) of Definition 4.7 does not require anything.

- If $\operatorname{UNFOLD}\left(\sigma_{1}\right)=$ ? $\mathrm{t}_{1} \cdot \sigma_{1}^{\prime}$ we have to show that

$$
\operatorname{UNFOLD}\left(\sigma_{2}\right)=? \mathrm{t}_{2} \cdot \sigma_{2}^{\prime}
$$

with $\mathrm{t}_{1} \preccurlyeq_{\mathrm{g}} \mathrm{t}_{2}$ and $\sigma_{1}^{\prime} \mathcal{R} \sigma_{2}^{\prime}$. An application of part (iii) of Lemma 5.2 shows that $\operatorname{UNFOLD}\left(\mathcal{M}^{-1}\left(\sigma_{1}\right)\right)=?\left[\mathrm{t}_{1}\right] ; \mathcal{M}^{-1}\left(\sigma_{1}^{\prime}\right)$. The fact that $\mathcal{M}^{-1}\left(\sigma_{1}\right) \preccurlyeq_{\text {ST }}$ $\mathcal{M}^{-1}\left(\sigma_{2}\right)$ let us use Definition 2.7 to deduce that $\mathcal{M}^{-1}\left(\sigma_{2}\right)=$ ? $\left[\mathrm{t}_{2}\right] ; . \mathcal{M}^{-1}\left(\sigma_{2}^{\prime}\right)$ for some $\mathrm{t}_{2}$ such that $\mathrm{t}_{1} \preccurlyeq \mathrm{g} \mathrm{t}_{2}$ and some $\mathcal{M}^{-1}\left(\sigma_{2}^{\prime}\right)$ such that $\mathcal{M}^{-1}\left(\sigma_{1}^{\prime}\right) \preccurlyeq_{\mathrm{ST}}$ $\mathcal{M}^{-1}\left(\sigma_{2}^{\prime}\right)$. From the last inequality and the definition of $\mathcal{R}$ it follows that $\sigma_{1}^{\prime} \mathcal{R} \sigma_{2}^{\prime}$. Since we have proven the conditions on the input actions $t_{1}$, $\mathrm{t}_{2}$ and on the continuations $\sigma_{1}^{\prime}, \sigma_{2}^{\prime}$ we have left only to show that the structure of $\operatorname{UNFOLD}\left(\sigma_{2}\right)$ is the required one; this follows from another application of part (iii) of Lemma 5.2

The other cases are analogous and left to the reader.

Corollary 5.8. The relation $\sqsubseteq^{\mathcal{S C}}$ is decidable.

Proof. To begin with, note that $\mathcal{M}$ is defined by structural induction, so it is decidable. The corollary then follows from Corollary 2 of GH05], which ensures that the relation $\preccurlyeq_{\mathrm{ST}}$ is decidable, and our Theorem 5.7, whereby we can prove the equality $\mathcal{M}\left(\preccurlyeq_{\mathrm{ST}}\right)=\sqsubseteq \mathcal{S C}$. 


\subsection{Examples and applications}

In this subsection we give a series of examples in order to discuss the results we obtained. The first two example are of theoretical nature, whereas the last two show some applications.

Example 5.9. [Type simulations and the weak simulation relation]

At this stage, a natural question arises, which concerns the relationship between type simulations and weak simulations Mil99. Assume the standard definition of the weak simulation [Mil99]; we use the symbol $\lesssim$ to denote the greatest weak simulation relation.

We begin by showing that, even though two session types are in a coinductive types simulation, their images through $\mathcal{M}$ need not be in a weak simulation. Consider the relation

$$
\mathcal{R}=\left\{\left(\oplus\left\langle\mathrm{I}_{1}: \mathrm{END}, \mathrm{I}_{2}: \mathrm{END}\right\rangle,\left(\oplus\left\langle\mathrm{I}_{1}: \mathrm{END}\right\rangle\right),(\mathrm{END}, \mathrm{END})\right\}\right.
$$

The standard co-inductive proof technique let one prove that the relation $\mathcal{R}$ is a type simulation. On the other hand, the definition of $\mathcal{M}$ implies that

$$
\begin{aligned}
\mathcal{M}\left(\oplus\left\langle\mathrm{l}_{1}: \mathrm{END}, \mathrm{l}_{2}: \mathrm{END}\right\rangle\right) & =! 1_{1} . \mathbf{1} \oplus ! \mathrm{I}_{2} . \mathbf{1} \\
\mathcal{M}\left(\oplus\left\langle\mathrm{l}_{1}: \mathrm{END}\right\rangle\right) & =! 1_{1} . \mathbf{1}
\end{aligned}
$$

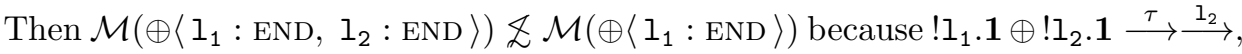
while $! 1_{1} .1 \stackrel{\jmath^{2}}{\gtrless^{*}}$. We have proven that $S_{1} \preccurlyeq{ }_{\text {ST }} S_{2}$ does not imply $\mathcal{M}\left(S_{1}\right) \lesssim$ $\mathcal{M}\left(S_{2}\right)$.

Looking at the foregoing argument, one might be tempted to reason that if $S_{1} \preccurlyeq_{\text {ST }} S_{2}$ then $\mathcal{M}\left(S_{2}\right) \lesssim \mathcal{M}\left(S_{1}\right)$. We prove that this is not the case. We can prove that

$$
? 1_{1} .1 \sqsubseteq^{\mathcal{S C}} ? 1_{2} \cdot 1+? 1_{1} \cdot 1
$$

An application of $\mathcal{M}^{-1}$ gives us:

$$
\begin{aligned}
\mathcal{M}^{-1}\left(? \mathrm{I}_{1} . \mathbf{1}\right) & =\&\left\langle\mathrm{I}_{1}: \mathrm{END}\right\rangle \\
\mathcal{M}^{-1}\left(? \mathrm{I}_{2} . \mathbf{1}+? \mathrm{I}_{1} . \mathbf{1}\right) & =\&\left\langle\mathrm{I}_{1}: \mathrm{END}, \mathrm{I}_{2}: \mathrm{END}\right\rangle
\end{aligned}
$$

A look at the definition of $\preccurlyeq_{\mathrm{ST}}$, Definition 2.7. lets one prove that for every type simulation $\mathcal{R}$

$$
\left(\&\left\langle l_{1}: \operatorname{END}, I_{2}: \operatorname{END}\right\rangle, \&\left\langle l_{1}: \operatorname{END}\right\rangle\right) \notin \mathcal{R}
$$

and, therefore,

$$
\&\left\langle I_{1}: \mathrm{END}, l_{2}: \operatorname{END}\right\rangle \aleph_{\mathrm{ST}} \&\left\langle l_{1}: \mathrm{END}\right\rangle
$$

Example 5.10. [e-vote, revisited]

In this example we use Theorem 5.7 in conjunction with Theorem 2 of GH05, in order to show how the set based pre-order $\complement^{\mathcal{S C}}$ can be used to guarantee that a process $P_{a}$ can be safely replaced by a suitable process $P_{b}$.

Consider two contracts BallotA and BallotB such that Ballot $\sqsubseteq^{\mathcal{S C}}$ BallotB Let Ballot $A=\mathcal{M}^{-1}$ (BallotA) and Ballot $B=\mathcal{M}^{-1}$ (BallotB). From Theorem 5.7 it follows that

$$
\text { Ballot } A \preccurlyeq_{\text {sт }} \text { Ballot } B
$$


Let $\perp_{c}$ denote the coinductive duality relation defined as in Definition 9 of GH05. Suppose now that $\operatorname{BltSRvA}\left(x^{+}\right), \operatorname{BltSRvB}\left(x^{+}\right)$and $\operatorname{Voter}\left(x^{-}\right)$are pi calculus processes (as in GH05]) such that

$$
\begin{array}{rll}
\left\{x^{+}: \text {Ballot } A\right\} & \vdash & \operatorname{BltSRvA}\left(x^{+}\right), \\
\left\{x^{+}: \text {BallotB }\right\} & \vdash & \operatorname{BltSRvB}\left(x^{+}\right), \\
\left\{x^{-}: \text {Voter }\right\} & \vdash & \operatorname{Voter}\left(x^{-}\right)
\end{array}
$$

for some session type Voter such that $V$ oter $\perp_{c}$ Ballot $A$. By means of the typing rules of GH05, it is possible to derive

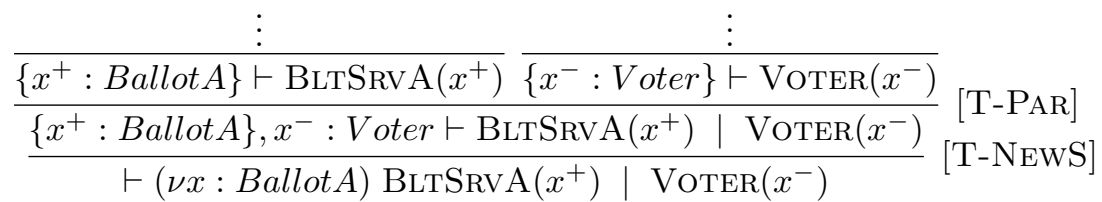

Then (10) above and Theorem 2 of GH05 can be used to guarantee that if process $\operatorname{BLtSRvB}\left(x^{+}\right)$is used in place of process $\operatorname{BLtSRv} \mathrm{A}\left(x^{+}\right)$, then no communication error will happen along the channel $x$.

One can use non-recursive versions of the contracts seen in Examples 3.13 and 4.4 to obtain contracts that satisfy the assumptions above:

$$
\begin{aligned}
& \text { BallotA = ?Login.(!Wrong.1 } \oplus \text { !Ok.( ?VoteA.1 + ?VoteB.1) } \\
& \text { BallotB = ?Login.('Wrong.1 } \oplus \\
& \text { !ok.( ?VoteA.1 + ?VoteB.1 + ?VoteC.1 + ?VoteD.1 )) } \\
& \text { Voter }=\mathcal{M}^{-1}(\text { !Login. }(\text { ?Wrong.1 }+ \text { ?Ok. }(\text { !VoteA.1 } \oplus ! \text { VoteB.1 })))
\end{aligned}
$$

Example 5.11. [Protocol conformace]

As already remarked, the language for contracts is a sublanguage of CCS without $\tau$ 's [NH87], and consequently contracts are suitable for specifying communication protocols.

Assume a protocol $\operatorname{Pr}$ to be specified by a contract $\sigma$, and let $Q$ be a process (in the sense of [GH05]), which is well-typed under the environment $\Gamma$. Assume also that $\Gamma(x)=S$ for some channel $x$.

We want to answer the following question:

(Q) "Does the session type $S$ conform to the protocol specification $\sigma$ ?"

Clearly, as long as the notion of conformance is not mathematically defined, it is not possible to give an answer (at least not a meaningful one).

In light of Theorem 5.7, we propose the following definition of conformance. Assume the standard definition of weak bisimilarity equivalence Mil99; we denote this relation $\approx$. We say that a session type $S$ conforms to a protocol specification $\sigma$ if and only if $\mathcal{M}(S) \approx \sigma$.

To answer the question $(\mathrm{Q})$ now one has only to prove that $\mathcal{M}(S) \approx \sigma$ or to show a counter example to this statement.

For example, if we had given a specification of the protocol POP3 Ros88 with a contract $\sigma$, then we would have been able to check whether the session type POP3 of GVR03 conforms to $\sigma$. 
In order for the notion of conformance we have given to be of any practical consequence, one last thing has to be ascertained. We have to prove that weak bisimilarity equivalence, when restricted to session contracts, is decidable. We leave this as an open problem worth further investigation.

\section{Conclusions}

\subsection{Summary}

In this paper we have used contracts CGP09 to give a fully abstract model for first-order session types ordered by their sub-typing relation GH05. This was achieved by identifying a subset of the standard language of contracts, CGP09, Pad10] which we call session contracts. These are ordered using a combination of two natural pre-orders Bd10, defined in terms of a contracts role in constraining the behaviours of servers and clients respectively.

The restriction to first-order session types is a severe limitation on our results. Despite this we believe that our work provides the first fully abstract model of session types in terms of contracts. We also intend to extend our results to the full language of session types in GH05. We claim that, contrary to what was done in Bd10, this can be achieved without using an higher-order LTS.

We have already stated in the Introduction that we use a subset of the language for contracts of $\mathrm{Pad10}$; nevertheless, the two languages are essentially the same, for the terms we lose are of no relevance for the theory. Our compliance relation coincides with the notion of strong compliance given there, although the formulation is different. Comparison with earlier work, LP07, LP08] is complicated by the fact that in these papers compliance judgements take the form $\mathrm{I}_{1}[\rho] \dashv \mathrm{I}_{2}[\sigma]$ where $\mathrm{I}_{1}, \mathrm{I}_{2}$ are finite sets of actions representing in some sense the interfaces of the processes guaranteeing the contracts; moreover, for a contract $\mathrm{I}[\sigma]$ to be valid its interface I has to contain all the action names that appear in the behaviour $\sigma$. Let us refer to these pairs $\mathrm{I}[\sigma]$ as constrained contracts. Using the obvious notation for the compliance relations between constrained contracts one can show if $\rho, \sigma$ are in $\mathcal{C}$ then

- if $\mathrm{I}[\rho] \dashv^{l p 07} \mathrm{~J}[\sigma]$ for some I, J then $\rho \dashv \sigma$

- If I $[\rho] \dashv^{l p 08} \mathrm{~J}[\sigma]$ for some I, J then $\rho \dashv \sigma$

under the assumption that in our definition of compliance the synchronisation relation $\bowtie_{i}$ is used. Moreover, it is easy to provide counter examples to the converse of both these points 4 We also believe that our result relating the server pre-order $\sqsubseteq_{\mathrm{SRV}}$ with the must-testing pre-order, Corollary 3.49 , is new, although a similar result is announced in Proposition 2.7 of $\mathrm{Pad10}$; however, there for a proof the reader is refered to [LP07] where the type of the compliance relation, and therefore the corresponding pre-order, the subcontract relation, differs from the type of the compliance of [Pad10.

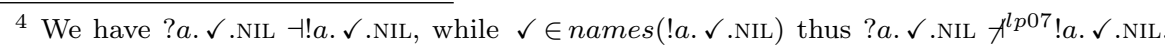
We also have ?a. $\checkmark$.NIL $+? b . \checkmark$. NIL $\dashv ! a . \checkmark$. NIL, and from $\{a, b\} \nsubseteq\{a\}$ it follows

$$
\{a, b, \checkmark\}[? a . \checkmark . \mathrm{NIL}+? b . \checkmark . \mathrm{NIL}]-y^{l p 08}\{a, \checkmark\}[! a \cdot \checkmark . \mathrm{NIL}] .
$$
}


Our research has been greatly influenced by the work in Bd10. In that paper the focus is the set of session behaviours, which is a proper subset of contracts and a proper superset of our session contracts; using this set the authors provide a sound model for sub-typing on session types. They use an interpretation function $\llbracket-\rrbracket$ from session types to session behaviours which, in general, is not invertible. For instance: ? Int.1 $+? \mathfrak{l}_{1} .1$ is a session behaviour that has no corresponding session type; this because Int is a base type while $l_{1}$ is a label. Note, though, that $\llbracket-\rrbracket=\mathcal{M}$, so the range of $\llbracket-\rrbracket$ is the set of session

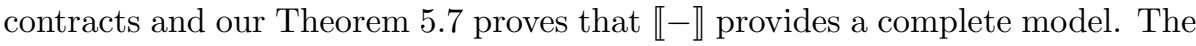
completeness of $\llbracket-\rrbracket$ was only conjectured in [Bd10].

Indeed, their approach is very similar to ours, in that they provide a coinductive characterisation of the intersection of the sub-server and sub-client pre-orders over session behaviours. In contrast, we have studied the individual pre-orders independently.

Finally in [P08 two interpretations, $\mathcal{M}_{\mathbf{1}}$ and $\mathcal{M}_{\mathrm{NIL}}$, similar to our $\mathcal{M}$, are given for pairs of session types into pairs of constrained contracts. Their proposed full abstraction result, Theorem 2 of [LP08, though, appears not to be true; what corresponds to our server pre-order in their paper is denoted by $\preceq$ and is defined in their Definition 2. According to that definition and the interpretation $\mathcal{M}_{\mathrm{NIL}}$

$$
\emptyset[\mathrm{NIL}] \preceq\{\ell\}[\ell . \mathrm{NIL}]
$$

Their Theorem 2 therefore implies $\&\langle\ell$ : END $\rangle \preccurlyeq_{\mathrm{ST}}$ END, which is obviously not true. On the other hand if $\mathcal{M}_{\mathbf{1}}$ is used then there are two issues. According to Theorem 2 the pair (END, $\&\langle\ell:$ END $\rangle)$ is interpreted as $(\emptyset[\checkmark . N I L],\{\ell\}[\ell . \checkmark . N I L])$. Then

(a) neither $\emptyset[\checkmark$. NIL $]$ nor $\{\ell\}[\ell . \checkmark$. NIL $]$ are constrained contracts, because their interfaces do not contain all the action names which appear in the respective behaviours; moreover

(b) even if the interpretation was correct, Theorem 2 would be false because

$$
\{\checkmark\}[\checkmark . \mathrm{NIL}] \preceq\{\ell, \checkmark\}[\ell . \checkmark . \mathrm{NIL}]
$$

while, as stated above, $\&\langle\ell:$ END $\rangle \preccurlyeq_{\mathrm{ST}}$ END is not true.

\section{References}

[ACKM04] Gustavo Alonso, Fabio Casati, Harumi A. Kuno, and Vijay Machiraju, Web services - concepts, architectures and applications, DataCentric Systems and Applications, Springer, 2004.

[BBMR08] Giovanni Bernardi, Michele Bugliesi, Damiano Macedonio, and Sabina Rossi, A theory of adaptable contract-based service composition, SYNASC (Viorel Negru, Tudor Jebelean, Dana Petcu, and Daniela Zaharie, eds.), IEEE Computer Society, 2008, pp. 327-334.

[Bd10] Franco Barbanera and Ugo de'Liguoro, Two notions of subbehaviour for session-based client/server systems, PPDP (Temur Kutsia, Wolfgang Schreiner, and Maribel Fernández, eds.), ACM, 2010, pp. 155-164. 
[BPZ09] Marco Bernardo, Luca Padovani, and Gianluigi Zavattaro (eds.), Formal methods for web services, 9th international school on formal methods for the design of computer, communication, and software systems, sfm 2009, bertinoro, italy, june 1-6, 2009, advanced lectures, Lecture Notes in Computer Science, vol. 5569, Springer, 2009 .

[CCLP06] Samuele Carpineti, Giuseppe Castagna, Cosimo Laneve, and Luca Padovani, A formal account of contracts for web services, WS-FM (Mario Bravetti, Manuel Núñez, and Gianluigi Zavattaro, eds.), Lecture Notes in Computer Science, vol. 4184, Springer, 2006, pp. 148 162.

[CGP09] Giuseppe Castagna, Nils Gesbert, and Luca Padovani, A theory of contracts for web services, ACM Trans. Program. Lang. Syst. 31 (2009), no. 5, 1-61, Supersedes the article in POPL '08.

[CP10] Luís Caires and Frank Pfenning, Session types as intuitionistic linear propositions, CONCUR (Paul Gastin and François Laroussinie, eds.), Lecture Notes in Computer Science, vol. 6269, Springer, 2010, pp. $222-236$.

[EF02] Rik Eshuis and Maarten M. Fokkinga, Comparing refinements for failure and bisimulation semantics, Fundam. Inform. 52 (2002), no. 4, 297-321.

[GH05] Simon J. Gay and Malcolm Hole, Subtyping for session types in the pi calculus, Acta Inf. 42 (2005), no. 2-3, 191-225.

[GVR03] Simon Gay, Vasco Vasconcelos, and Antonio Ravara, Session types for inter-process communication, Tech. Report TR-2003-133, Department of Computing Science, University of Glasgow, February 11 2003 .

[HVK98] Kohei Honda, Vasco Thudichum Vasconcelos, and Makoto Kubo, Language primitives and type discipline for structured communication-based programming, ESOP (Chris Hankin, ed.), Lecture Notes in Computer Science, vol. 1381, Springer, 1998, pp. 122 138.

[LP07] Cosimo Laneve and Luca Padovani, The must preorder revisited, Proceedings of the 18th international conference on Concurrency Theory (Berlin, Heidelberg), Springer-Verlag, 2007, pp. 212-225.

[LP08] Cosimo Laneve and Luca Padovani, The pairing of contracts and session types, Concurrency, Graphs and Models (Pierpaolo Degano, Rocco De Nicola, and José Meseguer, eds.), Lecture Notes in Computer Science, vol. 5065, Springer, 2008, pp. 681-700.

[Mil99] Robin Milner, Communicating and mobile systems - the pi-calculus, Cambridge University Press, 1999.

[NH84] Rocco De Nicola and Matthew Hennessy, Testing equivalences for processes, Theoretical Computer Science 34 (1984), 83-133. 
[NH87] _ CCS without $\tau$ 's, TAPSOFT, Vol.1, Lecture Notes in Computer Science, vol. 249, Springer, 1987, pp. 138-152.

[OasisS11] Oasis Standard, Universal Description, Discovery, and Integration, http://uddi.xml.org/, 2011.

[Pad10] Luca Padovani, Contract-based discovery of web services modulo simple orchestrators, Theor. Comput. Sci. 411 (2010), no. 37, 33283347.

[PS96] Benjamin C. Pierce and Davide Sangiorgi, Typing and subtyping for mobile processes, Mathematical Structures in Computer Science 6 (1996), no. 5, 409-453.

[Ros88] M.T. Rose, Post Office Protocol: Version 3, RFC 1081, November 1988, Obsoleted by RFC 1225. 